\title{
THE BOUNDARY COLLOCATION METHOD WITH MESHLESS CONCEPT FOR ACOUSTIC EIGENANALYSIS OF TWO-DIMENSIONAL CAVITIES USING RADIAL BASIS FUNCTION
}

\author{
J. T. Chen, M. H. Chang, K. H. Chen and S. R. Lin \\ Department of Harbor and River Engineering, National Taiwan Ocean University, 202 Keelung, \\ Taiwan, Republic of China. E-mail:jtchen@mail.ntou.edu.tw
}

(Received 30 July 2001, and in final form 8 January 2002)

\begin{abstract}
In this paper, a meshless method for the acoustic eigenfrequencies using radial basis function $(\mathrm{RBF})$ is proposed. The coefficients of influence matrices are easily determined by the two-point functions. In determining the diagonal elements of the influence matrices, two techniques, limiting approach and invariant method, are employed. Based on the RBF in the imaginary-part kernel, the method results in spurious eigenvalues which can be separated by using the singular value decomposition (SVD) technique in conjunction with the Fredholm alternative theorem. To understand why the spurious eigenvalues occur, analytical study in the discrete system by discretizing the circular boundary is conducted by using circulants. By using the SVD updating terms and documents, the true and spurious eigensolutions can be extracted out respectively. Several examples are demonstrated to see the validity of the present method.
\end{abstract}

(C) 2002 Elsevier Science Ltd. All rights reserved.

\section{INTRODUCTION}

The mesh generation of a complicated geometry is time consuming in the stage of model creation for engineers in dealing with the engineering problems by using the numerical methods, such as the finite difference method (FDM), finite element method (FEM) and boundary element method (BEM). In the recent years, researchers have paid attention to the meshless method which the element is free. The initial idea of meshless method dates back to the smooth particle hydrodynamics (SPH) method for modelling astrophysical phenomena [1]. Several meshless methods have also been reported in the literature, for example, the element-free Galerkin method [2] and the reproducing kernel method [3].

For acoustics, the integral equations have been utilized to solve the interior and exterior problems for a long time. Several approaches, e.g., complex-valued boundary element method [4], multiple reciprocity method (MRM) [5-7], and the real-part boundary element method $[5,8]$ have been developed for acoustic problems. To solve acoustic problems by using the complex-valued BEM, the influence coefficient matrix would be complex arithematics [9, 10]. Therefore, Tai and Shaw [11] employed only the real-part kernel to solve the eigenvalue problems and to avoid the complex-valued computation. The computation of the real-part kernel method or the MRM [11, 12] has some advantages, but it still faces both the singular and hypersingular integrals. To avoid the singular and hypersingular integrals, De Mey [13] used imaginary-part kernel to solve the eigenvalue 
problems. At the same time, De Mey also found the spurious eigensolutions but he did not discuss them analytically. Kang et al. [14-16] proposed the non-dimensional dynamic influence function (NDIF) method to solve the eigenproblem of an acoustic cavity. Later, Chen et al. [17] commented that the NDIF method is a special case of imaginary-part BEM. Nevertheless, spurious eigensolutions are inherent in the imaginary-part BEM, realpart BEM and MRM. Numerically speaking, the spurious eigensolutions result from the rank deficiency of the coefficient matrix which is less than $2 N$, where $2 N$ is the number of boundary unknowns. This implies the fewer number of constraint equations making the solution space larger. Mathematically speaking, the spurious eigensolutions for interior acoustics and fictitious solutions for exterior acoustics arise from an "improper approximation of the null space of operator".

In this paper, we will employ the imaginary-part kernel to solve the acoustic eigenproblems. In solving the problem numerically, elements are not required and only boundary nodes are necessary. Both the collocation and source points are distributed on the boundary only. Besides, the kernel function is composed of two-point function which is a kind of radial basis function. To examine the reason why spurious eigenvalues occur in the above methods, we will employ the SVD updating technique in conjunction with the Fredholm alternative theorem to overcome this difficulty by assembling the dual equations [18-20]. The SVD updating terms and updating documents will be employed to extract out the true and spurious eigenvalues for two-dimensional cavities respectively. For a special case of circular cavity, the spurious eigensolutions will be analytically predicted in the discrete system of circulants. Finally, the true eigenvalues for a circular cavity will be derived analytically by approaching the discrete system to the continuous system using the analytical properties of circulants [21].

\section{MESHLESS FORMULATION USING RADIAL BASIS FUNCTION OF THE IMAGINARY-PART KERNEL}

The governing equation for an interior acoustic problem is the Helmholtz equation as follows:

$$
\left(\nabla^{2}+k^{2}\right) u\left(x_{1}, x_{2}\right)=0, \quad\left(x_{1}, x_{2}\right) \in D,
$$

where $\nabla^{2}$ is the Laplacian operator, $D$ is the domain of the cavity and $k$ is the wave number which is angular frequency over the speed of sound. The boundary conditions can be either the Neumann or Dirichlet type.

The radial basis function is expressed by

$$
G\left(x_{i}, s_{j}\right)=\varphi\left(\left|s_{j}-x_{i}\right|\right),
$$

where $x_{i}$ and $s_{j}$ are the $i$ th collocation and $j$ th source points, respectively. The Euclidean norm $\left|s_{j}-x_{i}\right|$ is referred to as the radial distance between the collocation and source points. The two-point function $\left(\varphi\left(\left|s_{j}-x_{i}\right|\right)\right)$ is called radial basis function since it depends on the radial distance between $x_{i}$ and $s_{j}$. By considering the imaginary-part kernel of fundamental solution for the Helmholtz equation $\left(U(s, x)=\operatorname{Im}\left\{\mathrm{i} H_{0}^{(1)}(k r)\right\}\right)$ with globally supported radial basis function, we can choose the four kernels in the dual formulation $[5,22]$,

$$
\begin{gathered}
U(s, x)=\mathbf{J}_{0}(k|s-x|)=\mathbf{J}_{0}(k r), \\
T(s, x)=\frac{\partial U(s, x)}{\partial n_{s}}=-k \frac{\mathbf{J}_{1}(k r) y_{i} n_{i}}{r},
\end{gathered}
$$




$$
\begin{gathered}
L(s, x)=\frac{\partial U(s, x)}{\partial n_{x}}=k \frac{\mathrm{J}_{1}(k r) y_{j} \bar{n}_{j}}{r}, \\
M(s, x)=\frac{\partial^{2} U(s, x)}{\partial n_{s} \partial n_{x}}=k\left(\frac{-k \mathbf{J}_{2}(k r) y_{i} y_{j} n_{i} \bar{n}_{j}}{r^{2}}+\frac{\mathbf{J}_{1}(k r) n_{i} \bar{n}_{i}}{r}\right),
\end{gathered}
$$

where $r \equiv|s-x|$ is the distance between the source and collocation points; $n_{i}$ is the $i$ th component of the outnormal vector at $s ; \bar{n}_{i}$ is the $i$ th component of the outnormal vector at $x, \mathrm{~J}_{n}$ denotes the first-kind Bessel function of the $n$th order, and $y_{i} \equiv s_{i}-x_{i}, i=1,2$. Based on the dual formulation [23] for the indirect method, we can represent the acoustic field solution by

Single-layer potential approach:

$$
\begin{gathered}
u\left(x_{i}\right)=\sum_{j} U\left(s_{j}, x_{i}\right) \phi_{j} \underset{\text { matrix form }}{\rightarrow}\left\{u_{i}\right\}=\left[U_{i j}\right]\left\{\phi_{j}\right\}, \\
t\left(x_{i}\right)=\sum_{j} \frac{\partial U\left(s_{j}, x_{i}\right)}{\partial n_{x}} \phi_{j} \underset{\text { matrix form }}{\rightarrow}\left\{t_{i}\right\}=\left[L_{i j}\right]\left\{\phi_{j}\right\} .
\end{gathered}
$$

Double-layer potential approach:

$$
\begin{gathered}
u\left(x_{i}\right)=\sum_{j} T\left(s_{j}, x_{i}\right) \psi_{j} \underset{\text { matrix form }}{\rightarrow}\left\{u_{i}\right\}=\left[T_{i j}\right]\left\{\psi_{j}\right\}, \\
t\left(x_{i}\right)=\sum_{j} \frac{\partial T\left(s_{j}, x_{i}\right)}{\partial n_{x}} \psi_{j} \underset{\text { matrix form }}{\longrightarrow}\left\{t_{i}\right\}=\left[M_{i j}\right]\left\{\psi_{j}\right\},
\end{gathered}
$$

where $\left\{\phi_{j}\right\}$ and $\left\{\psi_{j}\right\}$ are the generalized unknowns by using the single- and double-layer potential approaches respectively. By adopting the two bases, $\mathbf{J}_{m}(k x)$ and its derivative $\mathbf{J}_{m}^{\prime}(k x)$, we can decompose the imaginary-part kernel functions into the separate forms,

$$
\begin{gathered}
U(s, x)= \begin{cases}U^{I}(\theta, 0)=\sum_{m=-\infty}^{\infty} \mathbf{J}_{m}(k R) \mathbf{J}_{m}(k \rho) \cos (m \theta), & R>\rho, \\
U^{E}(\theta, 0)=\sum_{m=-\infty}^{\infty} \mathbf{J}_{m}(k \rho) \mathbf{J}_{m}(k R) \cos (m \theta), & R<\rho,\end{cases} \\
T(s, x)= \begin{cases}T^{I}(\theta, 0)=\sum_{m=-\infty}^{\infty} k \mathbf{J}_{m}^{\prime}(k R) \mathbf{J}_{m}(k \rho) \cos (m \theta), & R>\rho, \\
T^{E}(\theta, 0)=\sum_{m=-\infty}^{\infty} k \mathbf{J}_{m}^{\prime}(k \rho) \mathbf{J}_{m}(k R) \cos (m \theta), & R<\rho,\end{cases} \\
L(s, x)= \begin{cases}L^{I}(\theta, 0)=\sum_{m=-\infty}^{\infty} k \mathbf{J}_{m}(k R) \mathbf{J}_{m}^{\prime}(k \rho) \cos (m \theta), & R>\rho, \\
L^{E}(\theta, 0)=\sum_{m=-\infty}^{\infty} k \mathbf{J}_{m}(k \rho) \mathbf{J}_{m}^{\prime}(k R) \cos (m \theta), & R<\rho,\end{cases} \\
M(s, x)= \begin{cases}M^{I}(\theta, 0)=\sum_{m=-\infty}^{\infty} k^{2} \mathbf{J}_{m}^{\prime}(k R) \mathbf{J}_{m}^{\prime}(k \rho) \cos (m \theta), & R>\rho, \\
M^{E}(\theta, 0)=\sum_{m=-\infty}^{\infty} k^{2} \mathbf{J}_{m}^{\prime}(k \rho) \mathbf{J}_{m}^{\prime}(k R) \cos (m \theta), & R<\rho,\end{cases}
\end{gathered}
$$

where the superscripts " $I$ " and " $E$ " denote the interior and exterior domains, $x=(\rho, 0)$ and $s=(R, \theta)$ in polar coordinate. By superimposing $2 N$ lumped density along the boundary, we have the four influence matrices

$$
\left[U_{i j}\right]=\left[\begin{array}{cccccc}
a_{1,1} & a_{1,2} & a_{1,3} & \cdots & a_{1,2 N-1} & a_{1,2 N} \\
a_{2,1} & a_{2,2} & a_{2,3} & \cdots & a_{2,2 N-1} & a_{2,2 N} \\
a_{3,1} & a_{3,2} & a_{3,3} & \cdots & a_{3,2 N-1} & a_{3,2 N} \\
\vdots & \vdots & \vdots & \ddots & \vdots & \vdots \\
a_{2 N, 1} & a_{2 N, 2} & a_{2 N, 3} & \cdots & a_{2 N, 2 N-1} & a_{2 N, 2 N}
\end{array}\right],
$$




$$
\begin{aligned}
{\left[T_{i j}\right]=} & {\left[\begin{array}{cccccc}
b_{1,1} & b_{1,2} & b_{1,3} & \cdots & b_{1,2 N-1} & b_{1,2 N} \\
b_{2,1} & b_{2,2} & b_{2,3} & \cdots & b_{2,2 N-1} & b_{2,2 N} \\
b_{3,1} & b_{3,2} & b_{3,3} & \cdots & b_{3,2 N-1} & b_{3,2 N} \\
\vdots & \vdots & \vdots & \ddots & \vdots & \vdots \\
b_{2 N, 1} & b_{2 N, 2} & b_{2 N, 3} & \cdots & b_{2 N, 2 N-1} & b_{2 N, 2 N}
\end{array}\right], } \\
{\left[L_{i j}\right]=} & {\left[\begin{array}{cccccc}
c_{1,1} & c_{1,2} & c_{1,3} & \cdots & c_{1,2 N-1} & c_{1,2 N} \\
c_{2,1} & c_{2,2} & c_{2,3} & \cdots & c_{2,2 N-1} & c_{2,2 N} \\
c_{3,1} & c_{3,2} & c_{3,3} & \cdots & c_{3,2 N-1} & c_{3,2 N} \\
\vdots & \vdots & \vdots & \ddots & \vdots & \vdots \\
c_{2 N, 1} & c_{2 N, 2} & c_{2 N, 3} & \cdots & c_{2 N, 2 N-1} & c_{2 N, 2 N}
\end{array}\right], } \\
{\left[M_{i j}\right]=} & {\left[\begin{array}{cccccc}
d_{1,1} & d_{1,2} & d_{1,3} & \cdots & d_{1,2 N-1} & d_{1,2 N} \\
d_{2,1} & d_{2,2} & d_{2,3} & \cdots & d_{2,2 N-1} & d_{2,2 N} \\
d_{3,1} & d_{3,2} & d_{3,3} & \cdots & d_{3,2 N-1} & d_{3,2 N} \\
\vdots & \vdots & \vdots & \ddots & \vdots & \vdots \\
d_{2 N, 1} & d_{2 N, 2} & d_{2 N, 3} & \cdots & d_{2 N, 2 N-1} & d_{2 N, 2 N}
\end{array}\right], }
\end{aligned}
$$

where the elements can be obtained by

$$
\begin{array}{ll}
a_{i, j}=U\left(s_{j}, x_{i}\right), & b_{i, j}=T\left(s_{j}, x_{i}\right), \\
c_{i, j}=L\left(s_{j}, x_{i}\right), & d_{i, j}=M\left(s_{j}, x_{i}\right) .
\end{array}
$$

Then, we can determine $\left\{\phi_{j}\right\}$ and $\left\{\psi_{j}\right\}$ by satisfying the boundary conditions.

\section{CALCULATION FOR THE DIAGONAL ELEMENTS IN THE FOUR INFLUENCE MATRICES USING THE L'HÔPITAL'S RULE AND INVARIANT METHOD}

The diagonal elements in the influence matrices where the radial distance is zero $(r=0$ when $i=j$ ) can be solved by using the L'Hôpital's rule. Considering the asymptotic behavior and the recurrence relations of Bessel functions, we can obtain the diagonal elements as follows:

$$
\begin{gathered}
\lim _{s \rightarrow x} U(s, x)=\lim _{r \rightarrow 0} \mathbf{J}_{0}(k r)=1, \\
\lim _{s \rightarrow x} T(s, x)=\lim _{r \rightarrow 0}-k \frac{\mathbf{J}_{1}(k r) y_{i} n_{i}}{r} \\
=\lim _{\substack{s_{1} \rightarrow x_{1} \\
s_{2} \rightarrow x_{2}}}-k^{2} \frac{\left(\mathbf{J}_{0}(k r)-\mathbf{J}_{2}(k r)\right) y_{i} n_{i}}{2}=0, \\
\lim _{s \rightarrow x} L(s, x)=\lim _{r \rightarrow 0} k \frac{\mathbf{J}_{1}(k r) y_{j} \bar{n}_{j}}{r} \\
=\lim _{\substack{s_{1} \rightarrow x_{1} \\
s_{2} \rightarrow x_{2}}} k^{2} \frac{\left(\mathbf{J}_{0}(k r)-\mathbf{J}_{2}(k r)\right) y_{j} \bar{n}_{j}}{2}=0,
\end{gathered}
$$




$$
\begin{aligned}
\lim _{s \rightarrow x} M(s, x) & =\lim _{r \rightarrow 0} k\left(\frac{-k \mathbf{J}_{2}(k r) y_{i} y_{j} n_{i} \bar{n}_{j}}{r^{2}}+\frac{\mathbf{J}_{1}(k r) n_{i} \bar{n}_{i}}{r}\right) \\
& =\lim _{\substack{s_{1} \rightarrow x_{1} \\
s_{2} \rightarrow x_{2}}} 0+k^{2} \frac{\left(\mathbf{J}_{0}(k r)-\mathbf{J}_{2}(k r)\right) n_{i} \bar{n}_{i}}{2}=\frac{k^{2}}{2},
\end{aligned}
$$

where $r=\sqrt{\left(s_{1}-x_{1}\right)^{2}+\left(s_{2}-x_{2}\right)^{2}}$.

For a circular case, the eigenvalues for the influence matrices in equations (15)-(18) can be obtained by

$$
\begin{aligned}
\lambda_{\ell} & =\frac{1}{\rho \triangle \theta} \int_{-\pi}^{\pi} \cos (\ell \theta) \sum_{m=-\infty}^{\infty} \mathbf{J}_{m}(k R) \mathbf{J}_{m}(k \rho) \cos (m \theta) \rho \mathrm{d} \theta \\
& =2 N \mathbf{J}_{\ell}(k \rho) \mathbf{J}_{\ell}(k \rho), \quad \ell=0, \pm 1, \ldots, \pm(N-1), N, \\
\mu_{\ell} & =\frac{1}{\rho \triangle \theta} \int_{-\pi}^{\pi} \cos (\ell \theta) \sum_{m=-\infty}^{\infty} \mathbf{J}_{m}^{\prime}(k R) \mathbf{J}_{m}(k \rho) \cos (m \theta) \rho \mathrm{d} \theta \\
& =2 N(k) \mathbf{J}_{\ell}^{\prime}(k \rho) \mathbf{J}_{\ell}(k \rho), \quad \ell=0, \pm 1, \ldots, \pm(N-1), N, \\
v_{\ell} & =\frac{1}{\rho \triangle \theta} \int_{-\pi}^{\pi} \cos (\ell \theta) \sum_{m=-\infty}^{\infty} \mathbf{J}_{m}(k R) \mathbf{J}_{m}^{\prime}(k \rho) \cos (m \theta) \rho \mathrm{d} \theta \\
& =2 N(k) \mathbf{J}_{\ell}(k \rho) \mathbf{J}_{\ell}^{\prime}(k \rho), \quad \ell=0, \pm 1, \ldots, \pm(N-1), N, \\
\kappa_{\ell} & =\frac{1}{\rho \triangle \theta} \int_{-\pi}^{\pi} \cos (\ell \theta) \sum_{m=-\infty}^{\infty} \mathbf{J}_{m}^{\prime}(k R) \mathbf{J}_{m}^{\prime}(k \rho) \cos (m \theta) \rho \mathrm{d} \theta \\
& =2 N\left(k^{2}\right) \mathbf{J}_{\ell}^{\prime}(k \rho) \mathbf{J}_{\ell}^{\prime}(k \rho), \quad \ell=0, \pm 1, \ldots, \pm(N-1), N,
\end{aligned}
$$

where $\triangle \theta=2 \pi / 2 N$. According to the addition theorem for the Bessel function and putting the same position for the two points, we have

$$
1=\mathbf{J}_{0}^{2}(k \rho)+2 \sum_{m=1}^{\infty} \mathbf{J}_{m}^{2}(k \rho) .
$$

By taking derivative with respect to $\rho$, we have

$$
0=\mathbf{J}_{0}(k \rho) \mathbf{J}_{0}^{\prime}(k \rho)+2 \sum_{m=1}^{\infty} \mathbf{J}_{m}(k \rho) \mathbf{J}_{m}^{\prime}(k \rho) .
$$

Using the invariant property for the influence matrices, the first invariant is the sum of all the eigenvalues

$$
\begin{aligned}
2 N a_{0} & =\lambda_{-(N-1)}+\cdots+\lambda_{-1}+\lambda_{0}+\lambda_{1}+\cdots+\lambda_{N} \\
& =2 N \sum_{\ell=-\infty}^{\infty} \mathbf{J}_{\ell}(k \rho) \mathbf{J}_{\ell}(k \rho), \\
2 N b_{0} & =\mu_{-(N-1)}+\cdots+\mu_{-1}+\mu_{0}+\mu_{1}+\cdots+\mu_{N} \\
& =2 N k \sum_{\ell=-\infty}^{\infty} \mathbf{J}_{\ell}^{\prime}(k \rho) \mathbf{J}_{\ell}(k \rho),
\end{aligned}
$$




$$
\begin{aligned}
2 N c_{0} & =v_{-(N-1)}+\cdots+v_{-1}+v_{0}+v_{1}+\cdots+v_{N} \\
& =2 N k \sum_{\ell=-\infty}^{\infty} \mathbf{J}_{\ell}(k \rho) \mathbf{J}_{\ell}^{\prime}(k \rho), \\
2 N d_{0} & =\kappa_{-(N-1)}+\cdots+\kappa_{-1}+\kappa_{0}+\kappa_{1}+\cdots+\kappa_{N} \\
& =2 N k^{2} \sum_{\ell=-\infty}^{\infty} \mathbf{J}_{\ell}^{\prime}(k \rho) \mathbf{J}_{\ell}^{\prime}(k \rho) .
\end{aligned}
$$

By substituting equations (31) and (32) into equations (33)-(36), we obtain $a_{0}=1, b_{0}=0$, $c_{0}=0$, and $d_{0}=k^{2} / 2$ if the imaginary-part kernel was considered. Hence, the indeterminate forms of diagonal elements are easily determined from the first invariant as well as the values obtained by using the L'Hôpital's rule.

\section{THE RELATIONS OF KERNELS AND MATRICES BETWEEN THE DIRECT AND INDIRECT METHODS}

In solving the boundary-value problem using BEM, two methods, direct method and indirect method are employed. The direct method is derived by using the Green identity in terms of the unknowns which are the actual physical quantities on the boundary. In the direct method, we have

$$
\begin{aligned}
& 0=\int_{B} T^{E}(s, x) u(s) \mathrm{d} B(s)-\int_{B} U^{E}(s, x) t(s) \mathrm{d} B(s), \\
& 0=\int_{B} M^{E}(s, x) u(s) \mathrm{d} B(s)-\int_{B} L^{E}(s, x) t(s) \mathrm{d} B(s),
\end{aligned}
$$

where $u(s)$ and $t(s)$ are the potential and its normal derivative on the boundary and $x$ is outside the domain.

A key point of the indirect method is to represent a solution which satisfies the governing equation. The unknown densities are determined by matching the boundary conditions. Based on the superposition principle for the potentials, we have

Single-layer potential approach:

$$
\begin{aligned}
& u(x)=\int_{B} U^{I}(s, x) \phi(s) \mathrm{d} B(s), \\
& t(x)=\int_{B} L^{I}(s, x) \phi(s) \mathrm{d} B(s) .
\end{aligned}
$$

Double-layer potential approach:

$$
\begin{aligned}
& u(x)=\int_{B} T^{I}(s, x) \psi(s) \mathrm{d} B(s), \\
& t(x)=\int_{B} M^{I}(s, x) \psi(s) \mathrm{d} B(s),
\end{aligned}
$$

where $\phi(s)$ and $\psi(s)$ are the single- and double-layer unknown densities, respectively. By discretizing equations (37) and (38), we have the linear algebraic equations for the direct method

$$
\left[T_{i j}^{E}\right]\left\{u_{j}\right\}=\left[U_{i j}^{E}\right]\left\{t_{j}\right\}
$$


TABLE 1

The distributed and concentrated-type of imaginary-part method for the single-and double-layer potential approaches

\begin{tabular}{|c|c|c|}
\hline & Distributed-type & Concentrated-type $^{\dagger}$ \\
\hline $\begin{array}{l}\text { Single-layer potential } \\
\text { approach }\end{array}$ & $\begin{array}{l}\text { Dirichlet problem: } \\
u(x)=\int_{B} U^{I}(s, x) \phi(s) \mathrm{d} B(s) \\
\text { Neumann problem: } \\
t(x)=\int_{B} L^{I}(s, x) \phi(s) \mathrm{d} B(s)\end{array}$ & $\begin{aligned} & \text { Dirichlet problem: } \\
u\left(x_{i}\right)= & \sum_{j} U^{I}\left(s_{j}, x_{i}\right) A_{j}=(S M)_{i j} A_{j} \\
& \text { Neumann problem: } \\
t\left(x_{i}\right)= & \sum_{j} L^{I}\left(s_{j}, x_{i}\right) A_{j}=\left(S M_{x}\right)_{i j} A_{j}\end{aligned}$ \\
\hline $\begin{array}{l}\text { Double-layer } \\
\text { potential approach }\end{array}$ & $\begin{array}{l}\text { Dirichlet problem: } \\
u(x)=\int_{B} T^{I}(s, x) \psi(s) \mathrm{d} B(s) \\
\text { Neumann problem: } \\
t(x)=\int_{B} M^{I}(s, x) \psi(s) \mathrm{d} B(s)\end{array}$ & $\begin{aligned} & \text { Dirichlet problem: } \\
u\left(x_{i}\right)= & \sum_{j} T^{I}\left(s_{j}, x_{i}\right) B_{j}=\left(S M_{s}\right)_{i j} B_{j} \\
& \text { Neumann problem: } \\
t\left(x_{i}\right)= & \sum_{j} M^{I}\left(s_{j}, x_{i}\right) B_{j}=\left(S M_{s x}\right)_{i j} B_{j}\end{aligned}$ \\
\hline
\end{tabular}

${ }^{\dagger}$ NDIF method by Kang et al. [14-16] is the special case.

$$
\left[M_{i j}^{E}\right]\left\{u_{j}\right\}=\left[L_{i j}^{E}\right]\left\{t_{j}\right\},
$$

where $\left\{u_{j}\right\}$ and $\left\{t_{j}\right\}$ are the potential and its normal derivative on the boundary $B$. By discretizing equations (39)-(42), we have the linear algebraic equations for the indirect method.

Single-layer potential approach:

$$
\begin{aligned}
& u\left(x_{i}\right)=\left[U_{i j}^{I}\right]\left\{\phi_{j}\right\}, \\
& t\left(x_{i}\right)=\left[L_{i j}^{I}\right]\left\{\phi_{j}\right\} .
\end{aligned}
$$

Double-layer potential approach:

$$
\begin{aligned}
& u\left(x_{i}\right)=\left[T_{i j}^{I}\right]\left\{\psi_{j}\right\}, \\
& t\left(x_{i}\right)=\left[M_{i j}^{I}\right]\left\{\psi_{j}\right\},
\end{aligned}
$$

where $\left\{\phi_{j}\right\}$ and $\left\{\psi_{j}\right\}$ are the single- and double-layer unknown densities, respectively. The distributed-type and concentrated-type of imaginary-part method for the single- and double-layer potential approaches are shown in Table 1. By considering the degenerate kernels equations (11)-(14) and comparing with equations (43)-(48), we can find the following relations between the interior and exterior kernels from equations (11)-(14), i.e.,

$$
\begin{gathered}
U_{i j}^{E}=U_{i j}^{I} \text { or } U^{E}(s, x)=U^{I}(x, s), \\
T_{i j}^{E}=L_{i j}^{I} \text { or } T^{E}(s, x)=L^{I}(x, s), \\
L_{i j}^{E}=T_{i j}^{I} \text { or } L^{E}(s, x)=T^{I}(x, s), \\
M_{i j}^{E}=M_{i j}^{I} \text { or } M^{E}(s, x)=M^{I}(x, s) .
\end{gathered}
$$


TABLE 2

SVD updating technique for the true and spurious eigensolutions for a circular cavity using the direct and indirect methods

\begin{tabular}{|c|c|c|c|c|c|c|c|}
\hline \multirow{2}{*}{$\begin{array}{l}\text { Boundary-value } \\
\text { problem }\end{array}$} & \multirow[t]{2}{*}{ Eigensolutions } & \multicolumn{2}{|c|}{ Density function } & \multicolumn{4}{|c|}{ True and spurious eigenvalues } \\
\hline & & Single-layer & Double-layer & Direct $\mathrm{m}$ & hod & Indirect & hod \\
\hline \multirow[t]{4}{*}{$\begin{array}{l}\text { Dirichlet } \\
\text { problem }\end{array}$} & True eigensolution & $\mathbf{J}_{m}(k \rho)=0$ & $\mathbf{J}_{m}(k \rho)=0$ & $\begin{array}{l}\text { SVD updating } \\
\text { term }\end{array}$ & {$\left[\begin{array}{l}U^{E} \\
L^{E}\end{array}\right]$} & $\begin{array}{l}\text { SVD updating } \\
\text { term }\end{array}$ & {$\left[\begin{array}{l}U^{I} \\
T^{I}\end{array}\right]$} \\
\hline & & (Figure 1(a)) & (Figure 1(b)) & & & & (Figure 1(c)) \\
\hline & Spurious eigensolution & $\mathbf{J}_{m}(k \rho)=0$ & $\mathbf{J}_{m}^{\prime}(k \rho)=0$ & $\begin{array}{c}\text { SVD updating } \\
\text { document }\end{array}$ & {$\left[\begin{array}{ll}L^{E} & M^{E}\end{array}\right]$} & $\begin{array}{l}\text { SVD updating } \\
\text { document }\end{array}$ & {$\left[\begin{array}{ll}T^{I} & M^{I}\end{array}\right]$} \\
\hline & & (Figure 1(a)) & (Figure 1(b)) & & & & (Figure $1(\mathrm{~d})$ ) \\
\hline \multirow[t]{4}{*}{$\begin{array}{l}\text { Neumann } \\
\text { problem }\end{array}$} & True eigensolution & $\mathbf{J}_{m}^{\prime}(k \rho)=0$ & $\mathbf{J}_{m}^{\prime}(k \rho)=0$ & $\begin{array}{l}\text { SVD updating } \\
\text { term }\end{array}$ & {$\left[\begin{array}{c}T^{E} \\
M^{E}\end{array}\right]$} & $\begin{array}{l}\text { SVD updating } \\
\text { term }\end{array}$ & {$\left[\begin{array}{c}L^{I} \\
M^{I}\end{array}\right]$} \\
\hline & & (Figure 2(a)) & (Figure 2(b)) & & & & (Figure 2(c)) \\
\hline & Spurious eigensolution & & $\mathbf{J}_{m}^{\prime}(k \rho)=0$ & $\begin{array}{c}\text { SVD updating } \\
\text { document }\end{array}$ & {$\left[\begin{array}{ll}U^{E} & T^{E}\end{array}\right]$} & $\begin{array}{l}\text { SVD updating } \\
\text { document }\end{array}$ & {$\left[\begin{array}{ll}U^{I} & L^{I}\end{array}\right]$} \\
\hline & & (Figure 2(a)) & (Figure 2(b)) & & & & (Figure 2(d)) \\
\hline
\end{tabular}

Note: $U^{I}=U^{E}, L^{I}=T^{E}, T^{I}=L^{E}$ and $M^{I}=M^{E}$. 


\section{DERIVATION OF TRUE AND SPURIOUS EIGENSOLUTIONS FOR THE CICULAR CAVITY USING THE DEGENERATE KERNELS}

In the circular cavity, we have the degenerate kernel functions in equations (11)-(14). We assume the single- and double-layer density functions on the boundary as

$$
\begin{aligned}
& \phi(s)=\sum_{n=-\infty}^{\infty}\left(A_{n} \cos (n \theta)+B_{n} \sin (n \theta)\right), \\
& \psi(s)=\sum_{n=-\infty}^{\infty}\left(C_{n} \cos (n \theta)+D_{n} \sin (n \theta)\right),
\end{aligned}
$$

where $A_{n}, B_{n}, C_{n}$ and $D_{n}$ are coefficients. For the Dirichlet problem, equation (39) reduced to

$$
\begin{aligned}
0 & =\int_{B} U^{I}(s, x) \phi(s) \mathrm{d} B(s) \\
& =\int_{0}^{2 \pi} \sum_{m=-\infty}^{\infty} \mathbf{J}_{m}(k R) \mathbf{J}_{m}(k \rho) \cos (m \theta) \sum_{n=-\infty}^{\infty}\left(A_{n} \cos (n \theta)+B_{n} \sin (n \theta)\right) \rho \mathrm{d} \theta,
\end{aligned}
$$

by using equation (53). By considering the orthogonality of trigonometric function, we have

$$
\begin{gathered}
\int_{0}^{2 \pi} \cos (m \theta) \cos (n \theta) \mathrm{d} \theta= \begin{cases}\pi, & m=n, \\
0, & m \neq n,\end{cases} \\
\int_{0}^{2 \pi} \cos (m \theta) \sin (n \theta) d \theta=0 .
\end{gathered}
$$

\begin{tabular}{|c|c|c|c|c|c|c|}
\hline Problem & Eigenvalues & 1 & 2 & 3 & 4 & 5 \\
\hline \multirow[t]{6}{*}{ Dirichlet problem } & $\mathbf{J}_{n}(k)$ & $2 \cdot 4042$ & $5 \cdot 5201$ & $8 \cdot 6537$ & 11.7915 & 14.9309 \\
\hline & $\mathbf{J}_{1}(k)$ & 3.8317 & 7.0156 & $10 \cdot 1735$ & $13 \cdot 3237$ & $16 \cdot 4706$ \\
\hline & $\mathrm{J}_{2}(k)$ & 5.1356 & 8.4172 & 11.6198 & 14.7959 & 17.9598 \\
\hline & $\mathbf{J}_{n}(k)$ & $6 \cdot 3802$ & $9 \cdot 7610$ & $13 \cdot 0152$ & 16.2234 & 19.4094 \\
\hline & $\mathrm{J}_{4}(k)$ & 7.5883 & 11.0647 & $14 \cdot 3725$ & $17 \cdot 6160$ & $20 \cdot 8269$ \\
\hline & $\mathbf{J}_{5}(k)$ & 8.7715 & $12 \cdot 3386$ & $15 \cdot 7002$ & 18.9801 & $22 \cdot 2178$ \\
\hline \multirow[t]{6}{*}{ Neumann problem } & $\mathbf{J}_{0}^{\prime}(k)$ & 0 & $3 \cdot 8317$ & 7.0156 & $10 \cdot 1735$ & $13 \cdot 3237$ \\
\hline & $\mathbf{J}_{1}^{\prime}(k)$ & 1.8412 & $5 \cdot 3314$ & 8.5363 & 11.7060 & 14.8636 \\
\hline & $\mathbf{J}_{2}^{\prime}(k)$ & 3.0542 & $6 \cdot 7071$ & $9 \cdot 9695$ & $13 \cdot 1703$ & $16 \cdot 3475$ \\
\hline & $\mathbf{J}_{3}^{\prime}(k)$ & $4 \cdot 2012$ & $8 \cdot 0152$ & $11 \cdot 3459$ & 14.5858 & $17 \cdot 7887$ \\
\hline & $\mathbf{J}_{4}^{\prime}(k)$ & $5 \cdot 3175$ & $9 \cdot 2824$ & $12 \cdot 6819$ & $15 \cdot 9641$ & $19 \cdot 1960$ \\
\hline & $\mathbf{J}_{5}^{\prime}(k)$ & $6 \cdot 4156$ & $10 \cdot 5199$ & $13 \cdot 9872$ & $17 \cdot 3128$ & 20.5755 \\
\hline
\end{tabular}

According to equation (56) and equation (57), equation (55) simplifies to

$$
\begin{aligned}
0 & =\int_{0}^{2 \pi} \sum_{m=-\infty}^{\infty} \mathbf{J}_{m}(k R) \mathbf{J}_{m}(k \rho) \cos (m \theta) \sum_{n=-\infty}^{\infty}\left(A_{n} \cos (n \theta)+B_{n} \sin (n \theta)\right) \rho \mathrm{d} \theta, \\
& =\pi \sum_{m=-\infty}^{\infty} A_{m} \mathbf{J}_{m}(k R) \mathbf{J}_{m}(k \rho) .
\end{aligned}
$$

\section{TABLE 3}

Zeros of the Bessel functions and its derivative, $\mathbf{J}_{n}(k)$ and $\mathbf{J}_{n}^{\prime}(k)$ 
Thus, we obtain the eigenequation $\mathbf{J}_{m}(k R) \mathbf{J}_{m}(k \rho)=0$ by using the single-layer potential methods for the interior Dirichlet problem.

Similarly, we also obtain

$$
\begin{aligned}
0 & =\int_{B} T^{I}(s, x) \psi(s) \mathrm{d} B(s) \\
& =\int_{0}^{2 \pi} \sum_{m=-\infty}^{\infty} k \mathbf{J}_{m}^{\prime}(k R) \mathbf{J}_{m}(k \rho) \cos (m \theta) \sum_{n=-\infty}^{\infty}\left(C_{n} \cos (n \theta)+D_{n} \sin (n \theta)\right) \rho \mathrm{d} \theta
\end{aligned}
$$

by using the double-layer potential approach. By considering equation (59) and the orthogonality of trigonometric function, we have

$$
\begin{aligned}
0 & =\int_{0}^{2 \pi} \sum_{m=-\infty}^{\infty} k \mathbf{J}_{m}^{\prime}(k R) \mathbf{J}_{m}(k \rho) \cos (m \theta) \sum_{n=-\infty}^{\infty}\left(C_{n} \cos (n \theta)+D_{n} \sin (n \theta)\right) \rho \mathrm{d} \theta \\
& =\pi k \sum_{m=-\infty}^{\infty} C_{m} \mathbf{J}_{m}^{\prime}(k R) \mathbf{J}_{m}(k \rho) .
\end{aligned}
$$

Thus, we obtain the eigenequation $\mathbf{J}_{m}^{\prime}(k R) \mathbf{J}_{m}(k \rho)=0$ by using the double-layer potential method for the interior Dirichlet problem.

In the Neumann problem, we have

$$
\begin{aligned}
0 & =\int_{B} L^{I}(s, x) \phi(s) \mathrm{d} B(s) \\
& =\int_{0}^{2 \pi} \sum_{m=-\infty}^{\infty} k \mathrm{~J}_{m}(k R) \mathbf{J}_{m}^{\prime}(k \rho) \cos (m \theta) \sum_{n=-\infty}^{\infty}\left(A_{n} \cos (n \theta)+B_{n} \sin (n \theta)\right) \rho \mathrm{d} \theta
\end{aligned}
$$

by substituting equations (13) and (53) into equation (40). By considering the orthogonality of trigonometric function, equation (61) simplifies to

$$
\begin{aligned}
0 & =\int_{0}^{2 \pi} \sum_{m=-\infty}^{\infty} k \mathbf{J}_{m}(k R) \mathbf{J}_{m}^{\prime}(k \rho) \cos (m \theta) \sum_{n=-\infty}^{\infty}\left(A_{n} \cos (n \theta)+B_{n} \sin (n \theta)\right) \rho \mathrm{d} \theta \\
& =\pi k \sum_{m=-\infty}^{\infty} A_{m} \mathbf{J}_{m}(k R) \mathbf{J}_{m}^{\prime}(k \rho) .
\end{aligned}
$$

Therefore, we obtain the eigenequation $\mathrm{J}_{m}(k R) \mathbf{J}_{m}^{\prime}(k \rho)=0$ by using the single-layer potential method for the interior Neumann problem.

Similarly, we use the double-layer density functions to obtain

$$
\begin{aligned}
0 & =\int_{B} M^{I}(s, x) \psi(s) \mathrm{d} B(s) \\
& =\int_{0}^{2 \pi} \sum_{m=-\infty}^{\infty} k^{2} \mathbf{J}_{m}^{\prime}(k R) \mathbf{J}_{m}^{\prime}(k \rho) \cos (m \theta) \sum_{n=-\infty}^{\infty}\left(C_{n} \cos (n \theta)+D_{n} \sin (n \theta)\right) \rho \mathrm{d} \theta .
\end{aligned}
$$

Figure 1. (a) The minimum singular value for different wave numbers by using the single-layer potential approach for the Dirichlet problem. (b) The minimum singular value for different wave numbers by using the double-layer potential approach for the Dirichlet problem. (c) The minimum singular value for different wave numbers using the SVD updating term $\left[\begin{array}{ll}U & T\end{array}\right]$ for the Dirichlet problem. (d) The minimum singular value for different wave numbers using the SVD updating document $\left[\begin{array}{ll}T & M\end{array}\right]$ for the Dirichlet problem. (e) The former three interior modes by using the single-layer potential approach for the Dirichlet problem. (f) The former three interior modes matrix by using the double-layer potential approach for the Dirichlet problem. $(\mathrm{g})$ The former three analytical interior modes for the Dirichlet problem. 

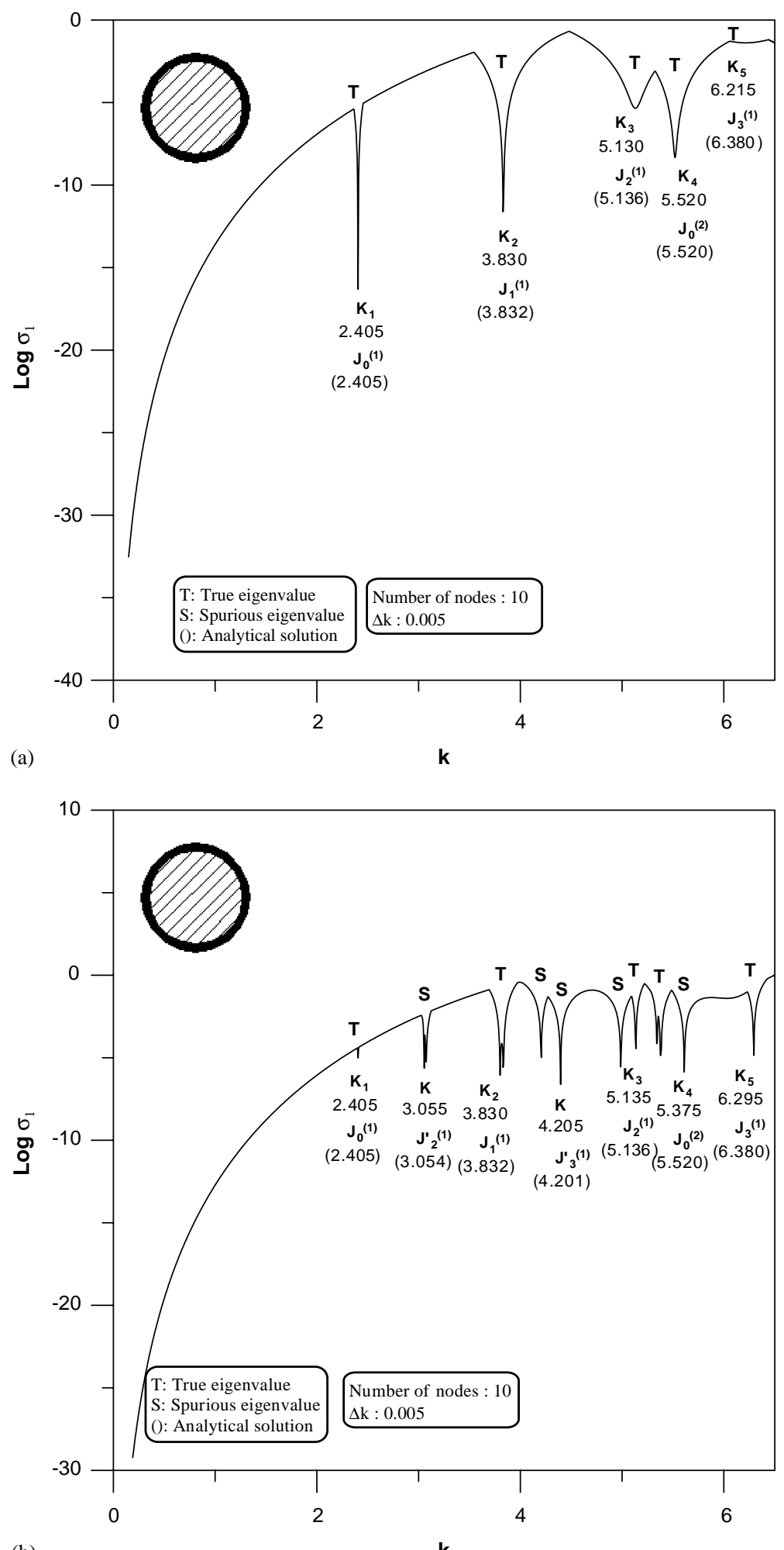

(b)

k 

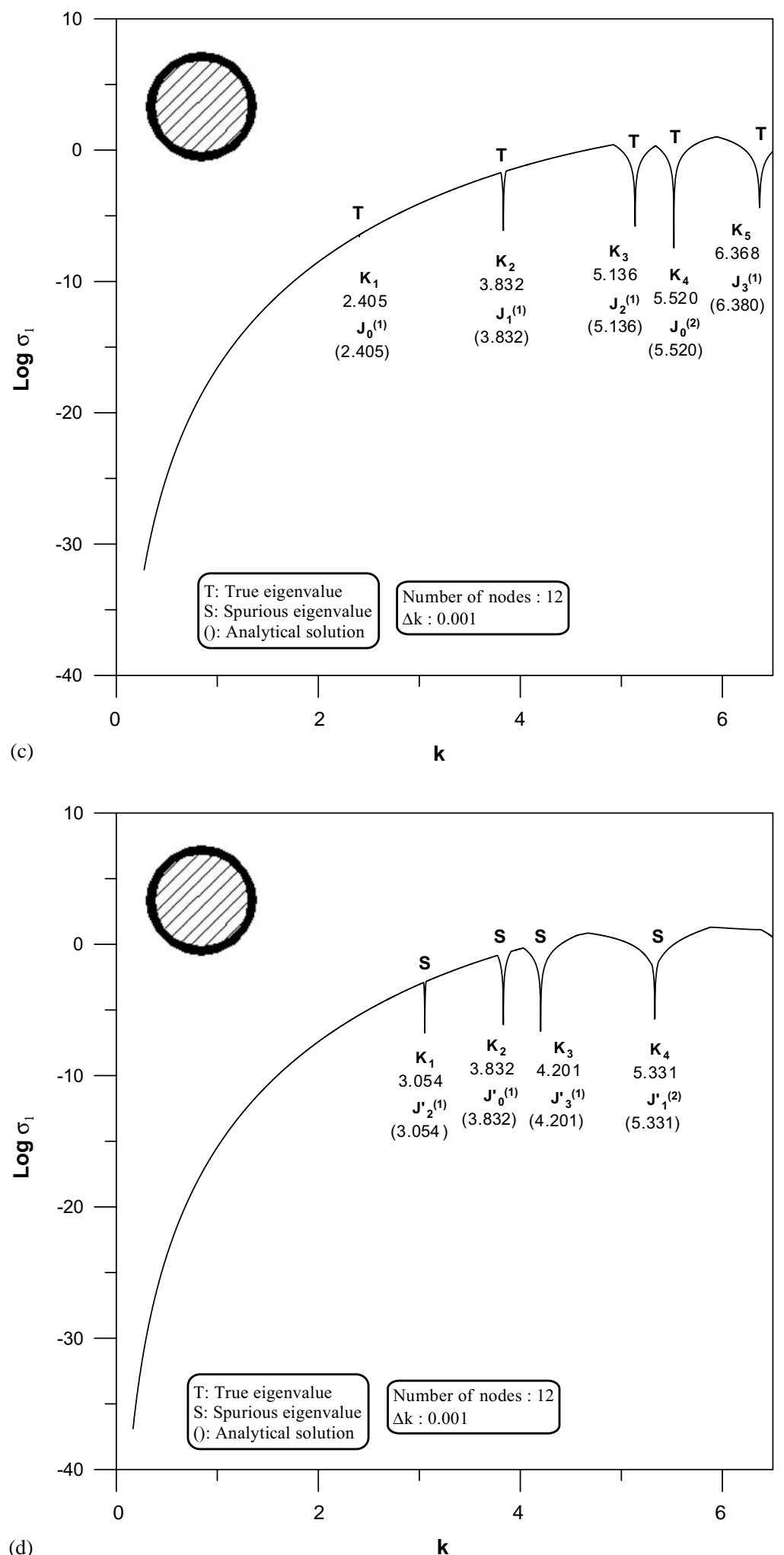

Figure 1. Continued. 
Mode $1(\mathrm{k}=2.405)$ (True)

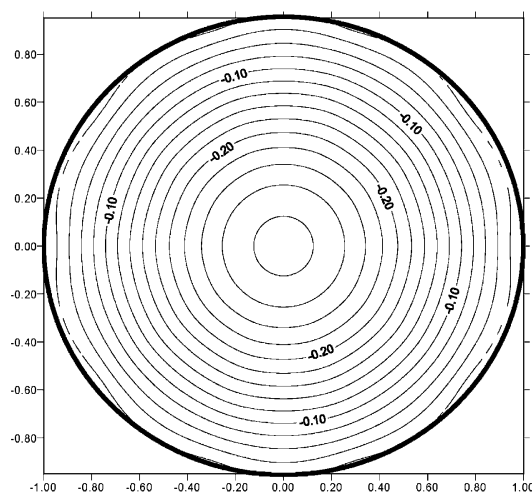

Mode $2(\mathrm{k}=3.830)$ (True)

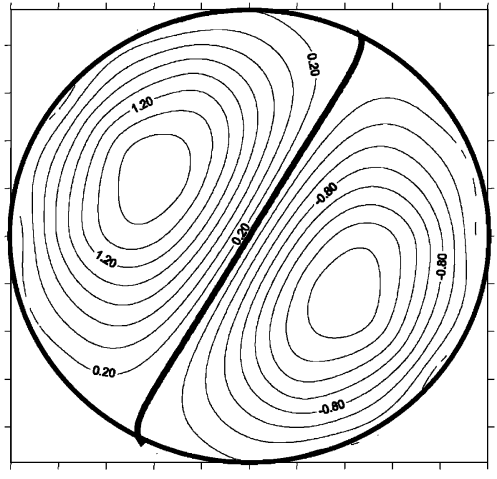

Mode $3(\mathrm{k}=5.130)$ (True)

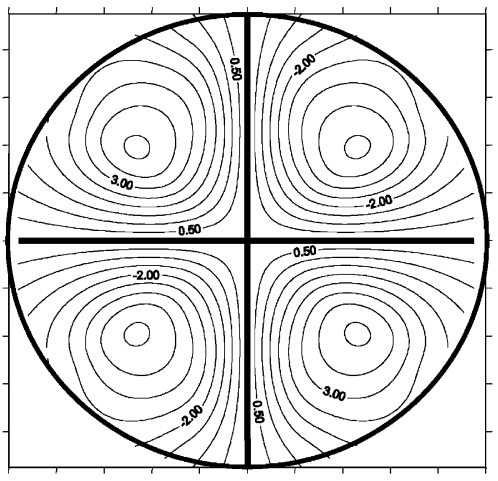

Mode $1(\mathrm{k}=2.405)$ (True)

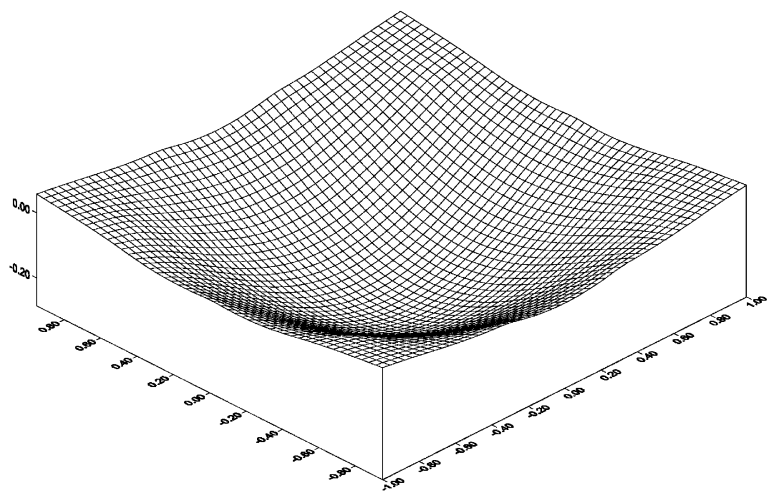

Mode $2(\mathrm{k}=3.830)$ (True)

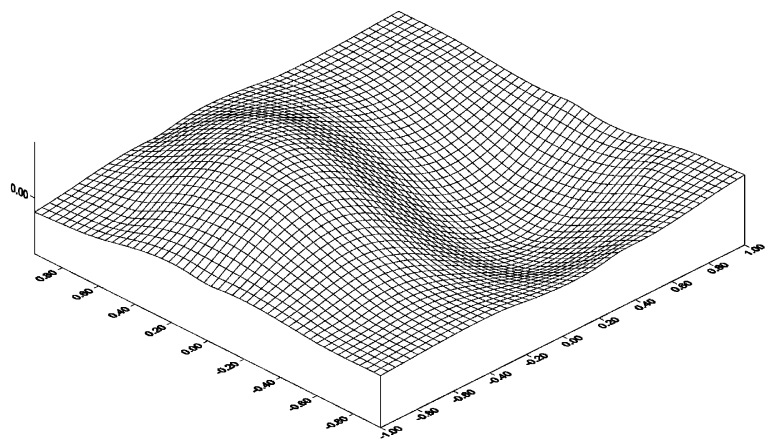

Mode $3(k=5.130)$ (True)

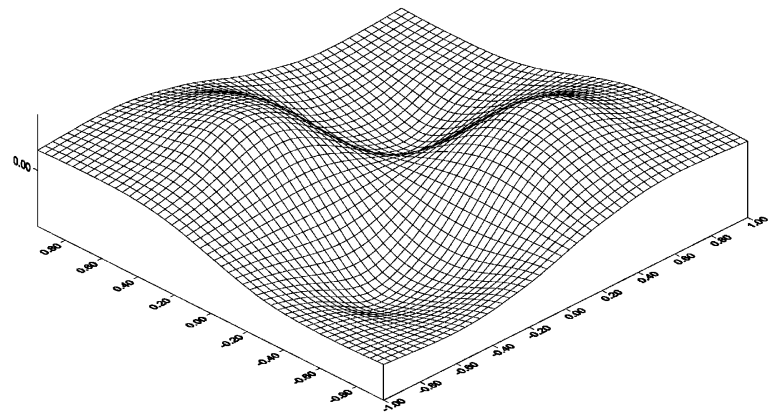

(e)

Figure 1. Continued. 

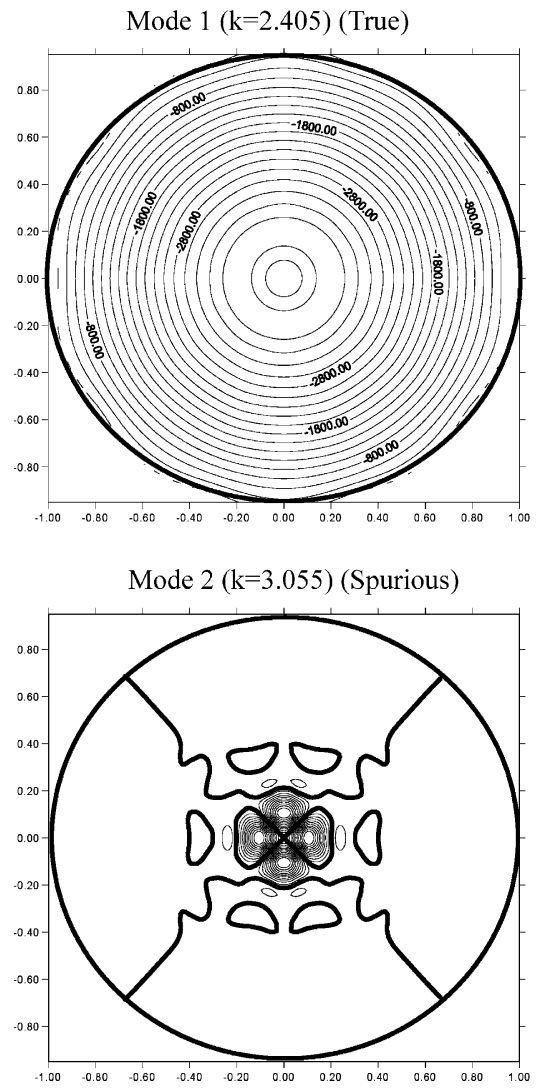

Mode $3(\mathrm{k}=3.830)$ (True)

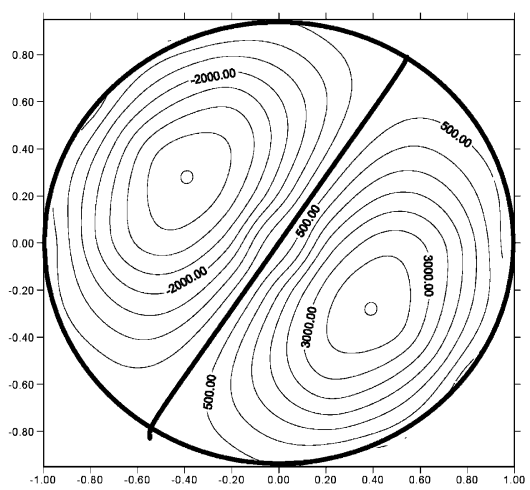

Mode $1(\mathrm{k}=2.405)$ (True)

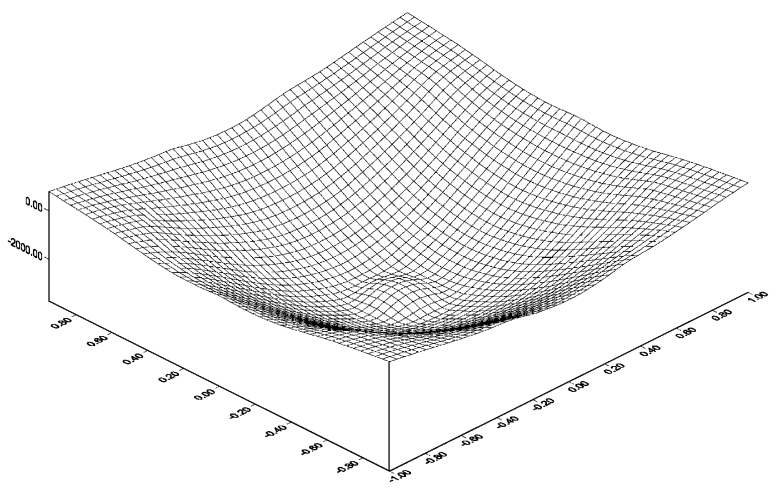

Mode $2(\mathrm{k}=3.055)$ (Spurious)

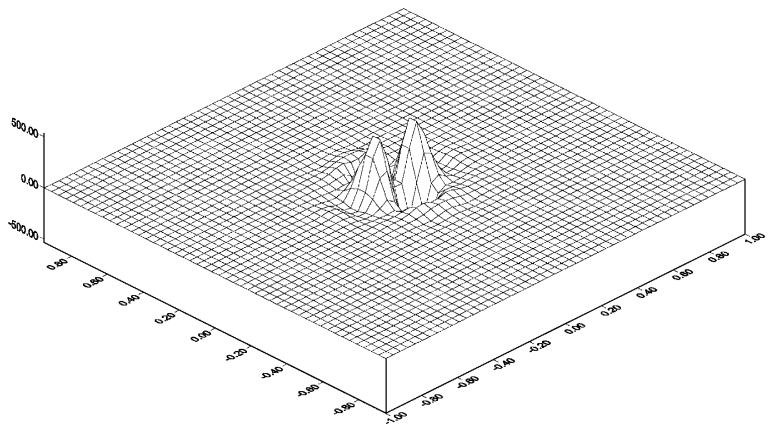

Mode $3(\mathrm{k}=3.830)$ (True)

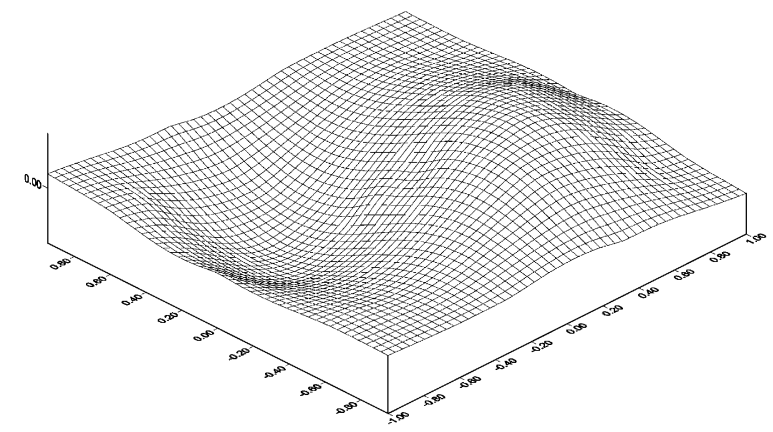

(f)

Figure 1. Continued. 
Mode $1(\mathrm{k}=2.404)$

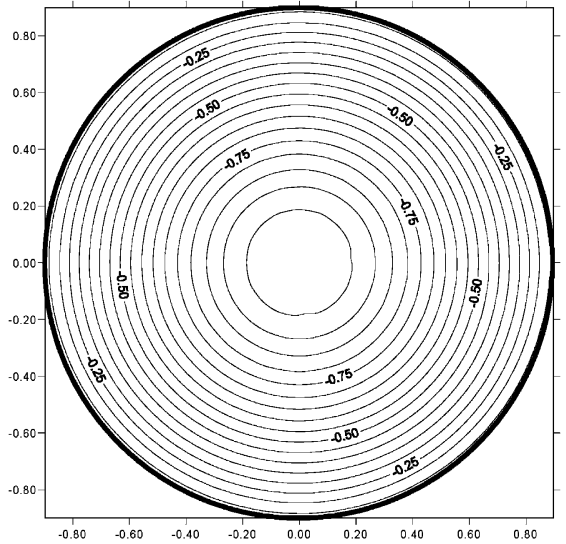

Mode $2(\mathrm{k}=3.832)$

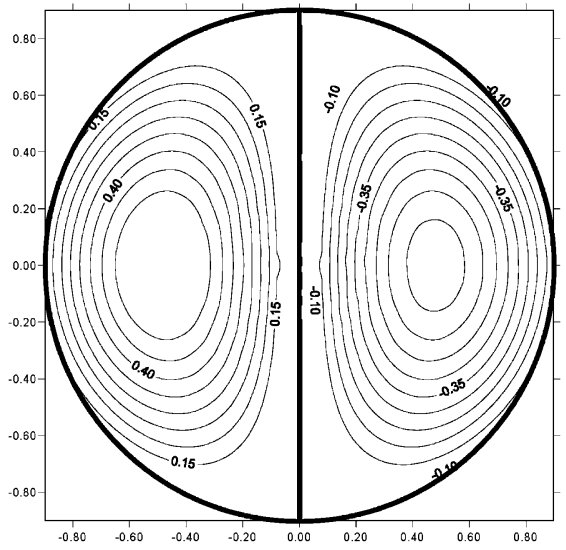

Mode $3(\mathrm{k}=5.136)$

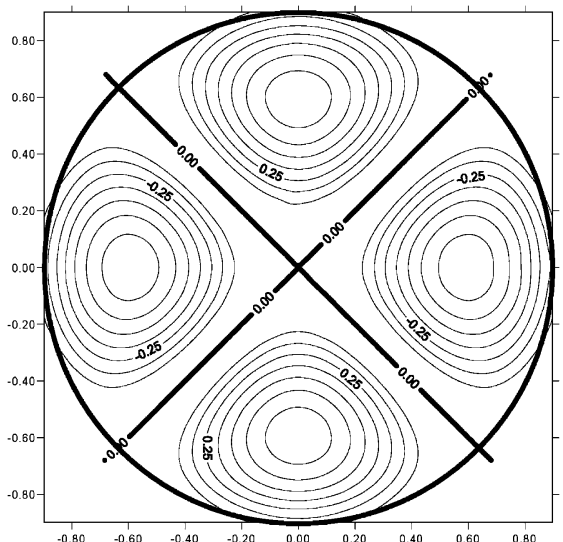

Mode $1(\mathrm{k}=2.404)$

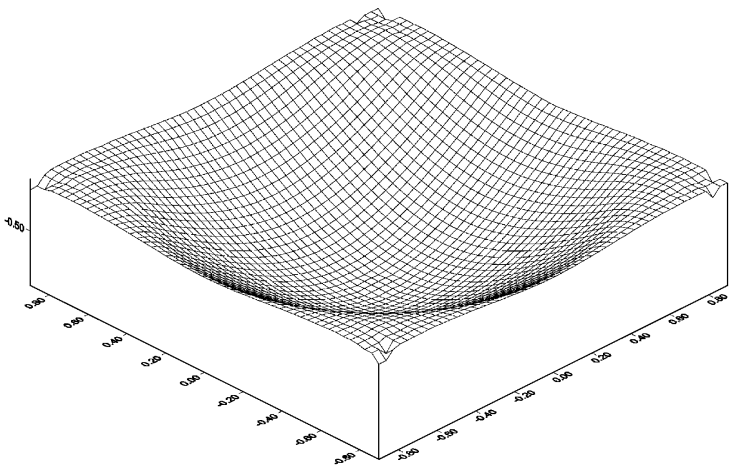

Mode $2(\mathrm{k}=3.832)$

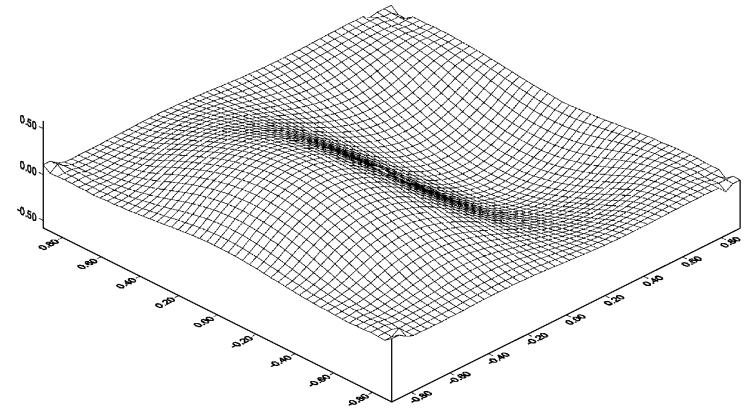

Mode $3(\mathrm{k}=5.136)$

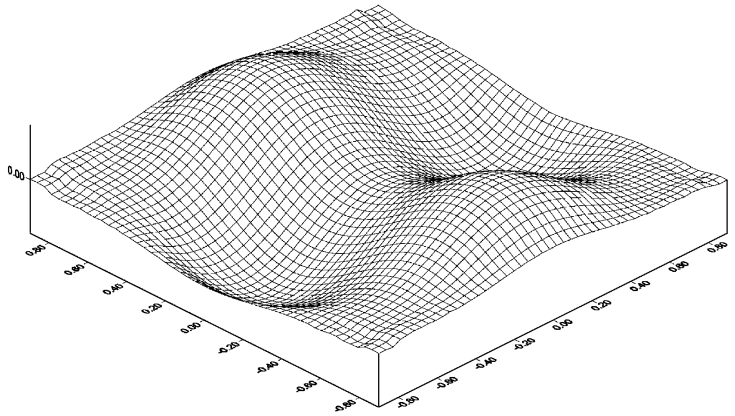

(g)

Figure 1. Continued. 
By considering equation (63) and the orthogonality of trigonometric function, we have

$$
\begin{aligned}
0 & =\int_{0}^{2 \pi} \sum_{m=-\infty}^{\infty} k^{2} \mathbf{J}_{m}^{\prime}(k R) \mathbf{J}_{m}^{\prime}(k \rho) \cos (m \theta) \sum_{n=-\infty}^{\infty}\left(C_{n} \cos (n \theta)+D_{n} \sin (n \theta)\right) \rho \mathrm{d} \theta \\
& =\pi k^{2} \sum_{m=-\infty}^{\infty} C_{m} \mathbf{J}_{m}^{\prime}(k R) \mathbf{J}_{m}^{\prime}(k \rho) .
\end{aligned}
$$

Therefore, we obtain the eigenequation $\mathbf{J}_{n}^{\prime}(k R) \mathbf{J}_{n}^{\prime}(k \rho)=0$ by using the double-layer potential method for the interior Neumann problem. All the relations of true and spurious eigenvalues by using the single- and double-layer potential approaches are summarized in Table 2.

\section{METHOD TO EXTRACT OUT THE TRUE EIGENSOLUTIONS}

The SVD technique is an important tool in linear algebra. A matrix $A$ with dimension $M \times N$ can be decomposed into a product of an orthogonal matrix $\Psi(N \times N)$, a diagonal matrix $\Sigma(M \times N)$ with positive or zero elements, and an orthogonal matrix $\Phi(M \times M)$,

$$
[A]_{M \times N}=[\Phi]_{M \times M}[\Sigma]_{M \times N}[\Psi]_{N \times N}^{\mathrm{T}}
$$

where the superscript " $T$ " is the transpose, $\Phi$ and $\Psi$ are both orthogonal in the sense that their column vectors are orthogonal,

$$
\begin{aligned}
& \phi_{i} \cdot \phi_{j}=\delta_{i j}, \\
& \psi_{i} \cdot \psi_{j}=\delta_{i j},
\end{aligned}
$$

where $\Phi^{\mathrm{T}} \Phi=\Psi^{\mathrm{T}} \Psi=I$ and $\delta_{i j}$ is the Kronecker delta symbol. Besides, we solve a homogeneous equation and obtain a non-trivial solution from a column vector $\left\{\psi_{i}\right\}$ of $\Psi$ such that the singular value $\left(\sigma_{i}\right)$ is zero. For the direct method in the discrete system, equations (43) and (44) are represented by

Singular equation:

$$
\text { (UT method) }\left[T^{E}\right]\{u\}=\left[U^{E}\right]\{t\}=0,
$$

Hypersingular equation:

$$
\text { (LM method) }\left[M^{E}\right]\{u\}=\left[L^{E}\right]\{t\}=0 .
$$

\section{TABLE 4}

True and spurious eigensolutions for the Dirichlet and Neumann problems using the present formulation

\begin{tabular}{lcc}
\hline \multicolumn{1}{c}{ Indirect formulation } & Dirichlet problem & Neumann problem \\
\hline $\begin{array}{l}\text { Single-layer potential approach } \\
\text { True mode }\end{array}$ & $-\pi \mathbf{J}_{n}(k a) \mathbf{J}_{n}(k \rho) \cos (n \phi)^{\dagger}$ & $-\pi k \mathbf{J}_{n}^{\prime}(k a) \mathbf{J}_{n}(k \rho) \cos (n \phi)$ \\
Spurious mode & $-\pi \mathbf{J}_{n}(k a) \mathbf{J}_{n}(k \rho) \cos (n \phi)^{\dagger}$ & $-\pi k \mathbf{J}_{n}^{\prime}(k a) \mathbf{J}_{n}(k \rho) \cos (n \phi)^{\dagger}$ \\
$\begin{array}{l}\text { Double-layer potential approach } \\
\text { True mode }\end{array}$ & $-\pi \mathbf{J}_{n}(k a) \mathbf{J}_{n}^{\prime}(k \rho) \cos (n \phi)$ & $-\pi k^{2} \mathbf{J}_{n}^{\prime}(k a) \mathbf{J}_{n}^{\prime}(k \rho) \cos (n \phi)^{\dagger}$ \\
Spurious mode & $-\pi \mathbf{J}_{n}(k a) \mathbf{J}_{n}^{\prime}(k \rho) \cos (n \phi)^{\dagger}$ & $-\pi k^{2} \mathbf{J}_{n}^{\prime}(k a) \mathbf{J}_{n}^{\prime}(k \rho) \cos (n \phi)^{\dagger}$ \\
\hline
\end{tabular}

Note: $0<a<\rho, 0<\phi<2 \pi$.

$\dagger$ Denotes a near-zero solution. 
For the Dirichlet problem, equations (68) and (69) are combined to

$$
\left[\begin{array}{c}
{\left[U^{E}\right]} \\
{\left[L^{E}\right]}
\end{array}\right]\{t\}=\{0\}
$$

By using the SVD technique, the two matrices in equation (70) are decomposed into

$$
\begin{gathered}
{\left[U^{E}\right]=\left[\Phi^{U}\right]\left[\Sigma^{U}\right]\left[\Psi^{U}\right]^{\mathrm{T}} \quad \text { or }\left[U_{j}^{E}\right]=\sum_{j} \sigma_{j}^{U}\left\{\phi_{j}^{U}\right\}\left\{\psi_{j}^{U}\right\}^{\mathrm{T}},} \\
{\left[L^{E}\right]=\left[\Phi^{L}\right]\left[\Sigma^{L}\right]\left[\Psi^{L}\right]^{\mathrm{T}} \quad \text { or }\left[L_{j}^{E}\right]=\sum_{j} \sigma_{j}^{L}\left\{\phi_{j}^{L}\right\}\left\{\psi_{j}^{L}\right\}^{\mathrm{T}} .}
\end{gathered}
$$

For the linear algebraic system, $t$ is one column vector $\left\{\psi_{i}\right\}$ in the $[\Psi]$ matrix corresponding to the zero singular value $\left(\sigma_{i}=0\right)$. By viewing $t$ as a $\left\{\psi_{i}\right\}$ vector in the right unitary matrix $[\Psi]$, equation $(70)$ reduces to

$$
\begin{aligned}
& {\left[U^{E}\right]\left\{\psi_{i}\right\}=\{0\},} \\
& {\left[L^{E}\right]\left\{\psi_{i}\right\}=\{0\},}
\end{aligned}
$$

since

$$
\begin{aligned}
& \sum_{j} \sigma_{j}^{U}\left\{\phi_{j}^{U}\right\}\left\{\psi_{j}^{U}\right\}^{\mathrm{T}}\left\{\psi_{i}\right\}=\{0\} \underset{\psi_{j}^{U} \cdot \psi_{j}=\delta_{i j}}{\rightarrow} \sigma_{i}^{U}\left\{\phi_{i}^{U}\right\}=\{0\} \quad(i \text { no sum }), \\
& \sum_{j} \sigma_{j}^{L}\left\{\phi_{j}^{L}\right\}\left\{\psi_{j}^{L}\right\}^{\mathrm{T}}\left\{\psi_{i}\right\}=\{0\} \underset{\psi_{j}^{L} \cdot \psi_{j}=\delta_{i j}}{\rightarrow} \sigma_{i}^{L}\left\{\phi_{i}^{L}\right\}=\{0\}, \quad(i \text { no sum }),
\end{aligned}
$$

where $\left\{\phi_{i}\right\}$ and $\left\{\psi_{i}\right\}$ are the orthonormal bases, $\sigma_{j}^{U}$ and $\sigma_{j}^{L}$ are the singular values of $\left[U^{E}\right]$ and $\left[L^{E}\right]$ matrices, respectively. We can easily extract out the true eigensolutions $\left(\sigma_{i}^{U}=\sigma_{i}^{L}=0\right)$ since there exists the same eigensolution $\left(t=\left\{\psi_{i}\right\}\right)$ in the Dirichlet problem using equations (70)-(73) together. In a similar way, equations (68) and (69) are combined to

$$
\left[\begin{array}{c}
{\left[T^{E}\right]} \\
{\left[M^{E}\right]}
\end{array}\right]\{u\}=\{0\}
$$

for the Neumann problem. We can easily extract the true eigensolutions;

$$
\begin{gathered}
\sum_{j} \sigma_{j}^{T}\left\{\phi_{j}\right\}\left\{\psi_{j}\right\}^{\mathrm{T}}\left\{\psi_{i}\right\}=\{0\} \underset{\psi_{i} \cdot \psi_{j}=\delta_{i j}}{\rightarrow} \sigma_{i}^{T}\left\{\phi_{i}\right\}=\{0\} \quad(i \text { no sum }), \\
\sum_{j} \sigma_{j}^{M}\left\{\phi_{j}\right\}\left\{\psi_{j}\right\}^{\mathrm{T}}\left\{\psi_{i}\right\}=\{0\} \underset{\psi_{i} \cdot \overrightarrow{\psi_{j}}=\delta_{i j}}{\rightarrow} \sigma_{i}^{M}\left\{\phi_{i}\right\}=\{0\} \quad(i \text { no sum }),
\end{gathered}
$$

since there exists the same solution $\left(u=\left\{\psi_{i}\right\}\right)$ corresponding to the zero singular values $\left(\sigma_{i}^{T}=\sigma_{i}^{M}=0\right)$ by using equations (74) and (75).

According to the relations between the direct method and indirect method (equations (49)-(52)), we can extend to extract out the true solutions in the indirect method. For the Dirichlet problem, equation (70) changes to

$$
\left[\begin{array}{c}
{\left[U^{E}\right]} \\
{\left[L^{E}\right]}
\end{array}\right]\{t\}=\{0\} \frac{U^{E}=U^{I}}{L^{E}=T^{T}}\left[\begin{array}{l}
{\left[U^{I}\right]} \\
{\left[T^{I}\right]}
\end{array}\right]\{t\}=\{0\},
$$

after using equations (49)-(51). By using the SVD technique and equation (76), we have

$$
\sum_{j} \sigma_{j}^{U}\left\{\phi_{j}\right\}\left\{\psi_{j}\right\}^{\mathrm{T}}\left\{\psi_{i}\right\}=\{0\} \underset{\psi_{i} \cdot \psi_{j}=\delta_{i j}}{\rightarrow} \sigma_{i}^{U}\left\{\phi_{i}\right\}=\{0\} \quad \text { (i no sum), }
$$




$$
\sum_{j} \sigma_{j}^{T}\left\{\phi_{j}\right\}\left\{\psi_{j}\right\}^{T}\left\{\psi_{i}\right\}=\{0\} \underset{\psi_{i} \cdot \psi_{j}=\delta_{i j}}{\rightarrow} \sigma_{i}^{T}\left\{\phi_{i}\right\}=\{0\} \quad(i \text { no sum }),
$$

where $\left\{\psi_{i}\right\}$ is an orthonormal basis; $\sigma_{j}^{U}$ and $\sigma_{j}^{T}$ are the singular values of $\left[U^{I}\right]$ and $\left[T^{I}\right]$ matrices, respectively. We can easily extract the true solutions in the Dirichlet problem by using equation (77) for the zero singular values of $\sigma_{i}^{U}=\sigma_{i}^{T}=0$.

In a similar way, equation (74) changes to

$$
\left[\begin{array}{c}
{\left[T^{E}\right]} \\
{\left[M^{E}\right]}
\end{array}\right]\{t\}=\{0\} \frac{T^{E}=L^{I}}{M^{E}=M^{I}}\left[\begin{array}{c}
{\left[L^{I}\right]} \\
{\left[M^{I}\right]}
\end{array}\right]\{t\}=\{0\},
$$

after using equations (50)-(52). By using the SVD technique and equation (78), we have

$$
\begin{aligned}
& \left.\sum_{j} \sigma_{j}^{L}\left\{\phi_{j}\right\}\left\{\psi_{j}\right\}^{\mathrm{T}}\left\{\psi_{i}\right\}=\{0\} \underset{\psi_{i} \psi_{j}=\delta_{i j}}{\rightarrow} \sigma_{i}^{L}\left\{\phi_{i}\right\}=\{0\} \quad \text { (i no sum }\right), \\
& \sum_{j} \sigma_{j}^{M}\left\{\phi_{j}\right\}\left\{\psi_{j}\right\}^{\mathrm{T}}\left\{\psi_{i}\right\}=\{0\} \underset{\psi_{i}{ }_{i} \psi_{j}=\delta_{i j}}{\longrightarrow} \sigma_{i}^{M}\left\{\phi_{i}\right\}=\{0\} \quad(i \text { no sum }),
\end{aligned}
$$

where $\left\{\psi_{i}\right\}$ is an orthonormal basis, $\sigma_{j}^{L}$ and $\sigma_{j}^{M}$ are the singular values of $\left[L^{I}\right]$ and $\left[M^{I}\right]$ matrices, respectively. We can easily extract out the true eigensolutions in the Neumann problem by using equation (79) for the zero singular values of $\sigma_{i}^{L}=\sigma_{i}^{M}=0$.

\subsection{EXTRACTING OUT THE TRUE EIGENSOLUTIONS BY USING THE SVD UPDATING TERM FOR A CIRCULAR CASE}

For a circular cavity subject to the Dirichlet boundary condition, we obtain the two eigenequations

$$
\begin{aligned}
& \text { Single-layer approach : } \mathbf{J}_{i}(k \rho) \mathbf{J}_{i}(k \rho)=0 \quad(i \text { no sum }), \\
& \text { Double-layer approach : } \mathbf{J}_{i}(k \rho) \mathbf{J}_{i}^{\prime}(k \rho)=0 \quad(i \text { no sum }) .
\end{aligned}
$$

The true eigensolution $\mathrm{J}_{i}(k \rho)=0$ satisfies both equations and the spurious eigensolutions $\mathbf{J}_{i}^{\prime}(k \rho)=0$ satisfies only one of the equations by using the double-layer potential approach for the Dirichlet problem.

To obtain an overdetermined system for the Dirchlet problem, we can combine $\left[U^{I}\right]$ and $\left[T^{I}\right]$ matrices by using the updating terms,

$$
[C]=\left[\begin{array}{l}
{\left[U^{I}\right]} \\
{\left[T^{I}\right]}
\end{array}\right]_{4 N \times 2 N} .
$$

Since the eigensolution is non-trivial, the rank of $[C]$ must be smaller than $2 N$. Therefore, the $2 N$ singular values for $[C]$ matrix must have at least one zero value. Based on the equivalence between the SVD technique and the least-squares method in the mathematical

Figure 2. (a) The minimum singular value for different wave numbers by using the single-layer potential approach for the Neumann problem. (b) The minimum singular value for different wave numbers by using the double-layer potential approach for the Neumann problem. (c) The minimum singular value for different wave numbers by using the SVD updating term $\left[\begin{array}{ll}L & M\end{array}\right]$ for the Neumann problem. (d) The minimum singular value for different wave numbers by using the SVD updating document $\left[\begin{array}{ll}U & L\end{array}\right]$ for the Neumann problem. (e) The former three interior modes by using the single-layer potential approach for the Neumann problem. (f) The former three interior modes by using the double-layer potential approach for the Neumann problem. (g) The former three analytical interior modes for the Neumann problem. 

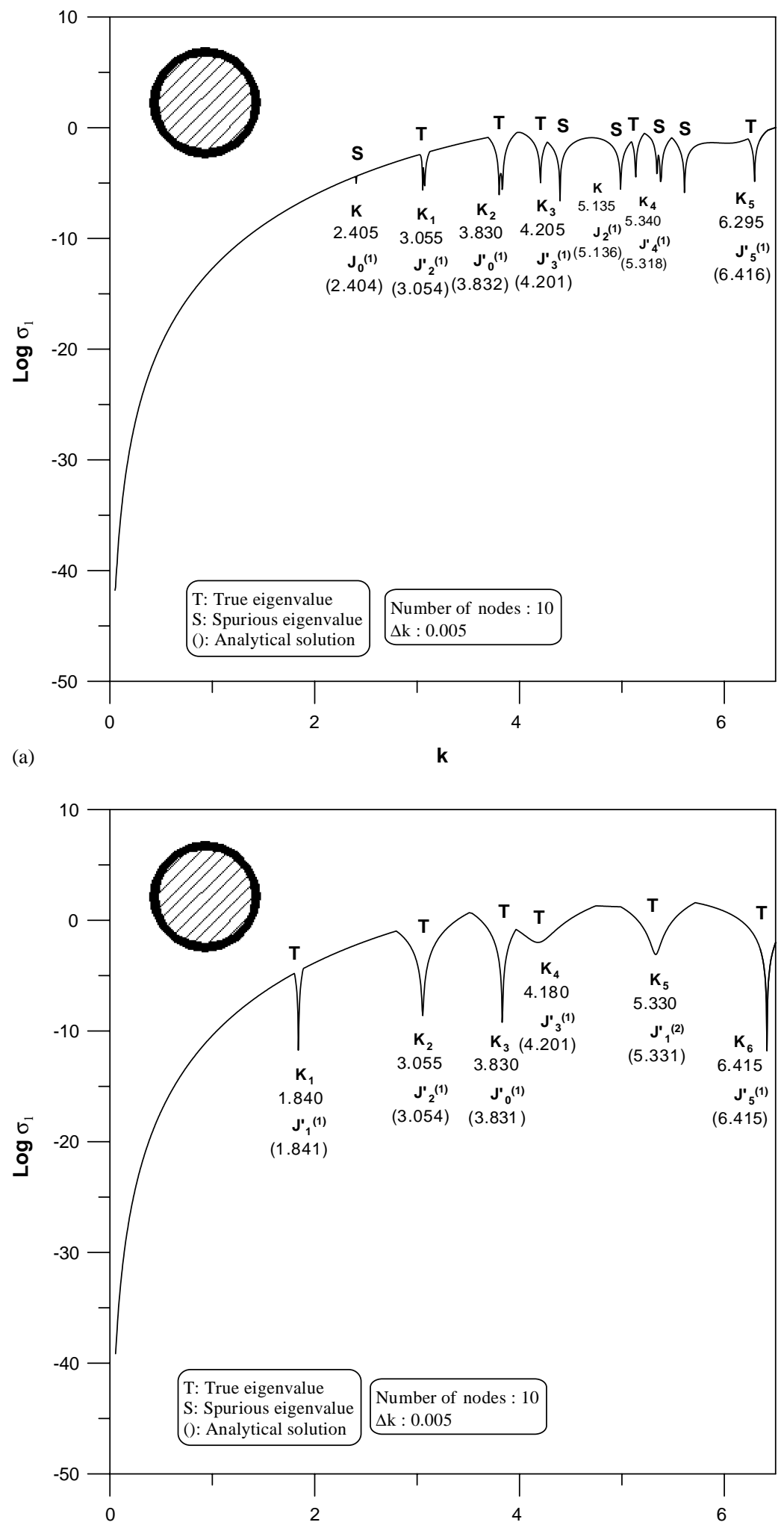

(b) 


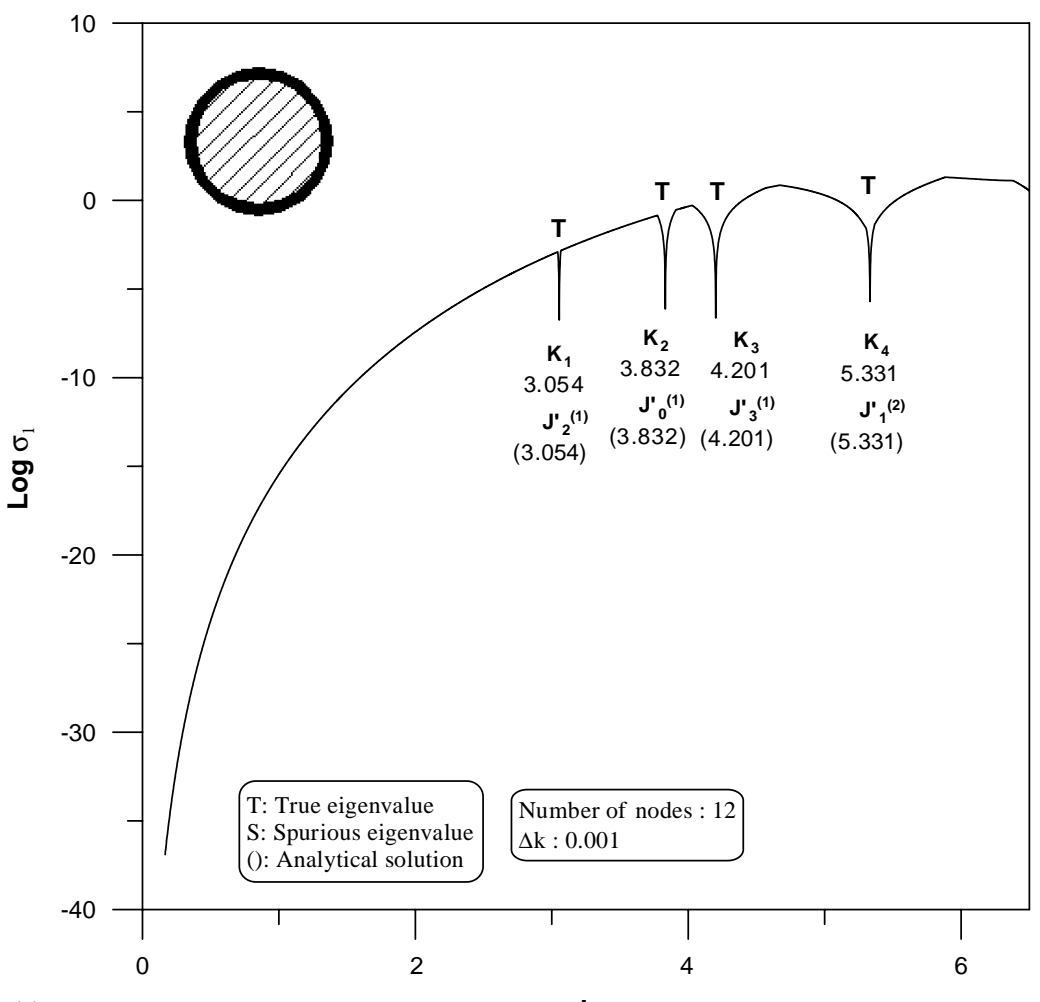

(c)

k

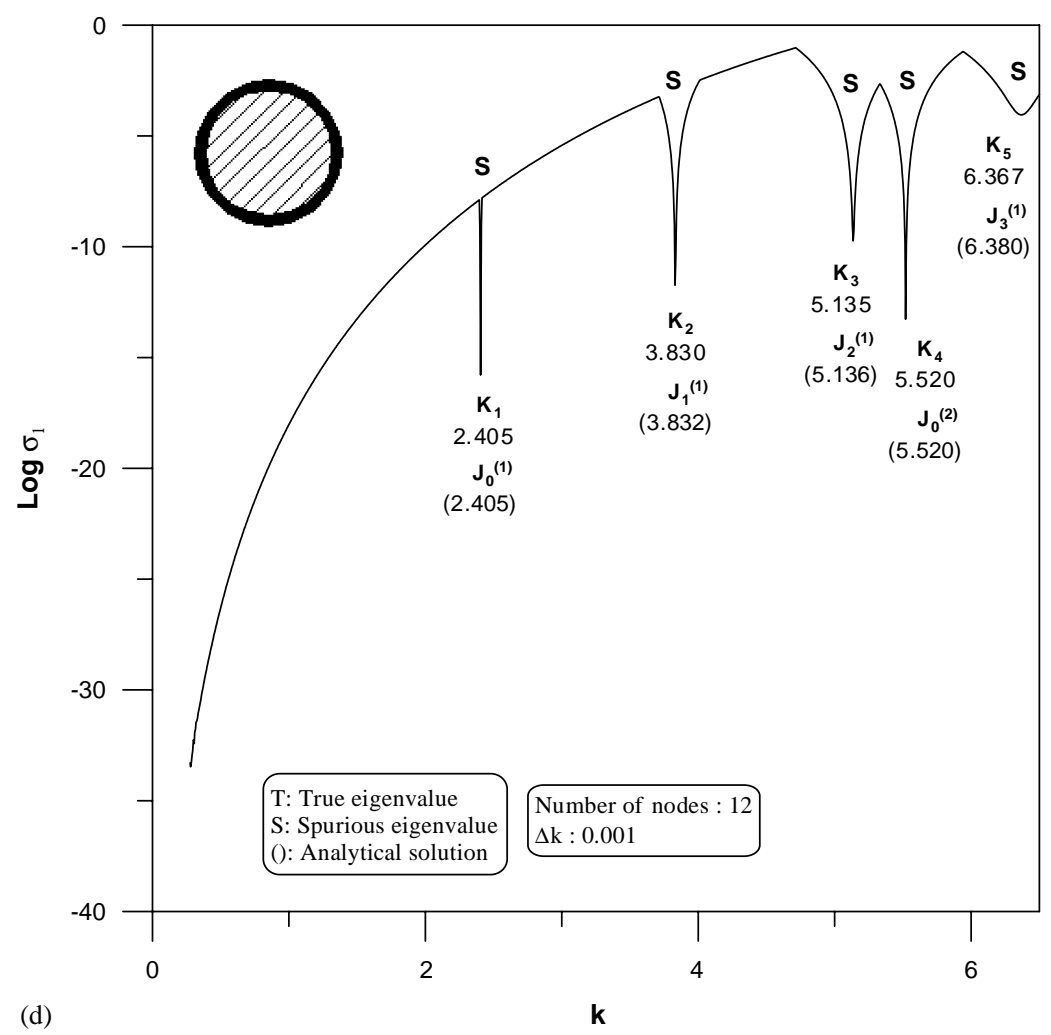

Figure 2. Continued. 
Mode $1(\mathrm{k}=2.405)$ (Spurious)

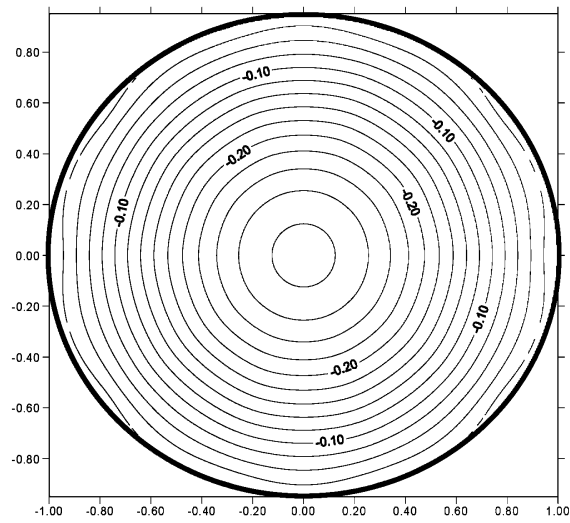

Mode $2(\mathrm{k}=3.055)$ (True)

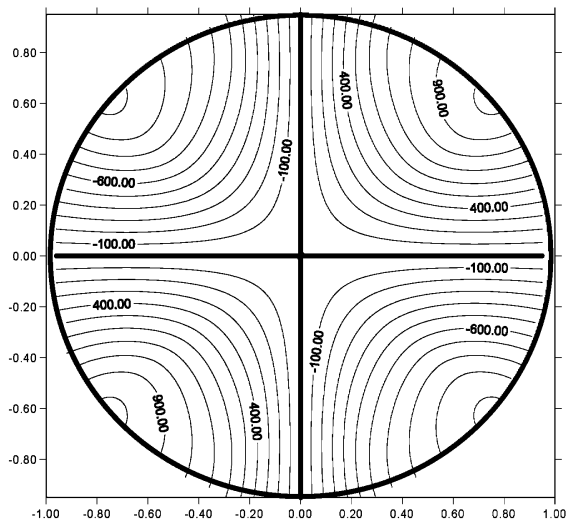

Mode $3(\mathrm{k}=3.830)$ (True)

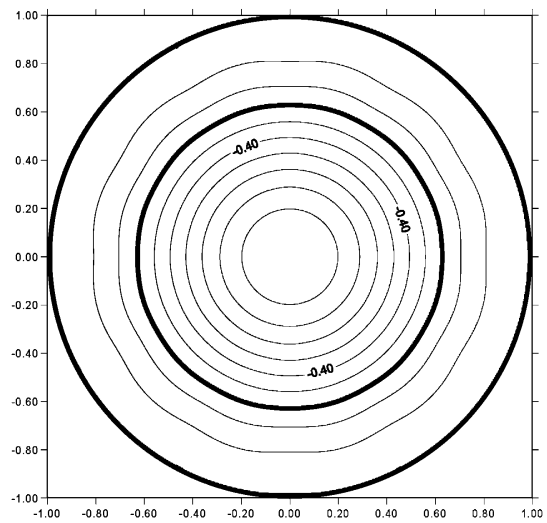

Mode $1(\mathrm{k}=2.405)$ (Spurious)

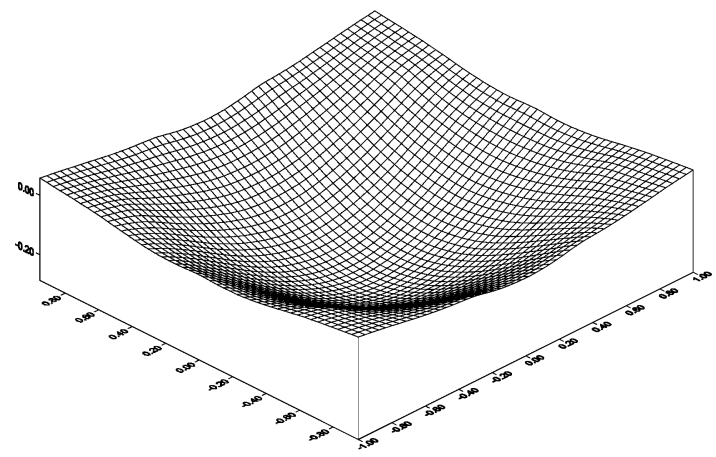

Mode $2(\mathrm{k}=3.055)$ (True)

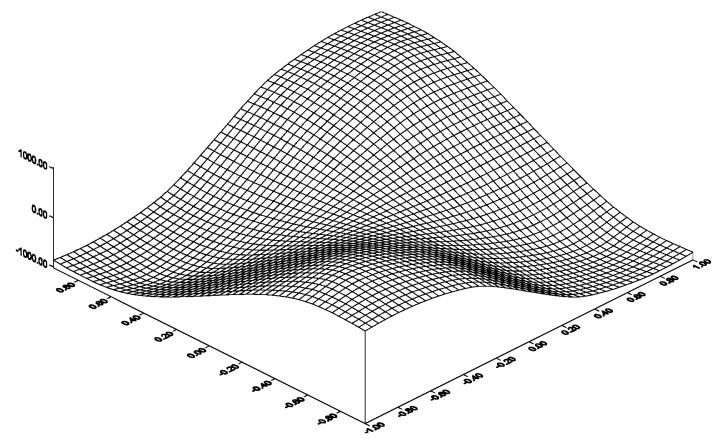

Mode $3(\mathrm{k}=3.830)$ (True)

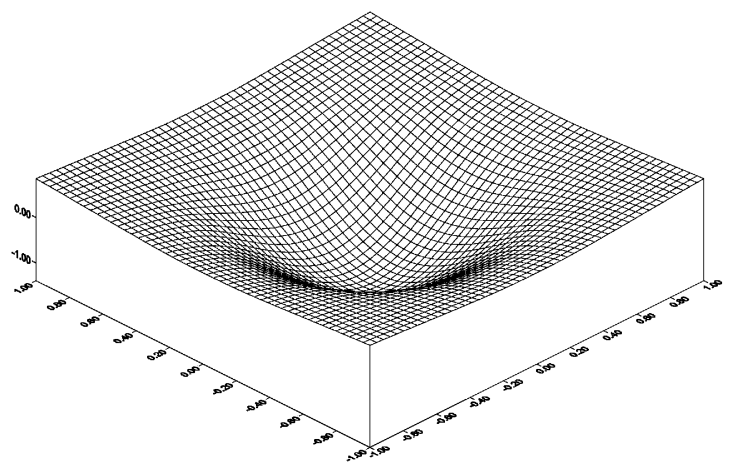

(e)

Figure 2. Continued. 

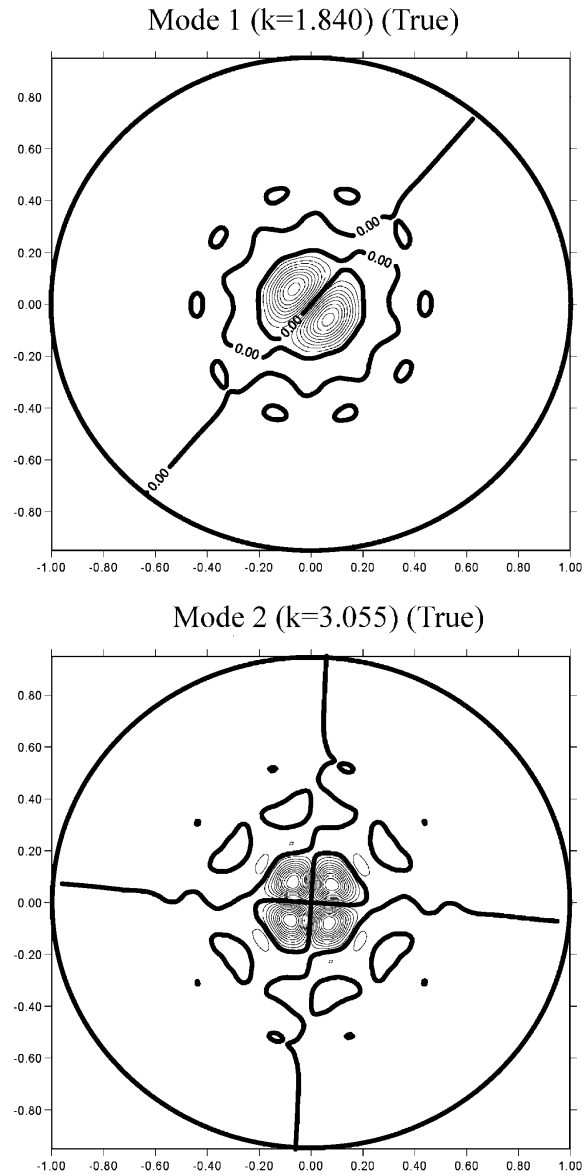

Mode $3(\mathrm{k}=3.830)$ (True)

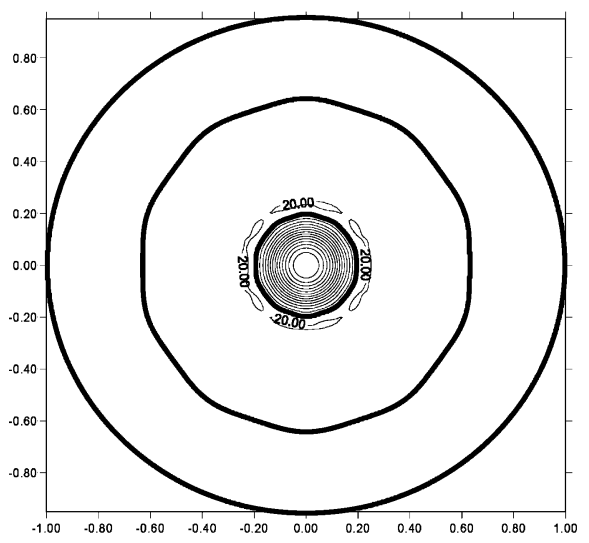

Mode $1(\mathrm{k}=1.840)($ True $)$

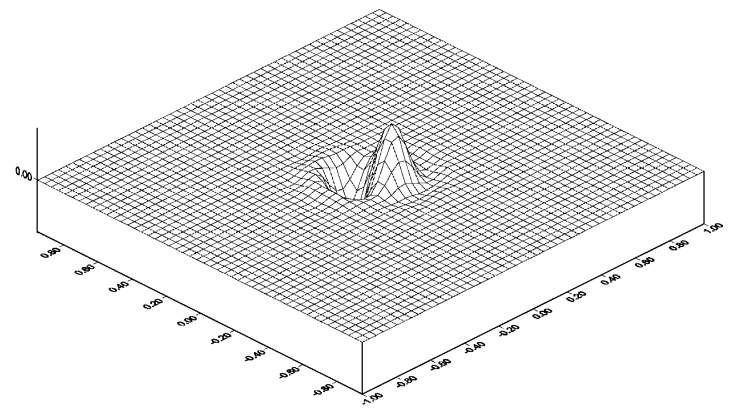

Mode $2(\mathrm{k}=3.055)$ (True)

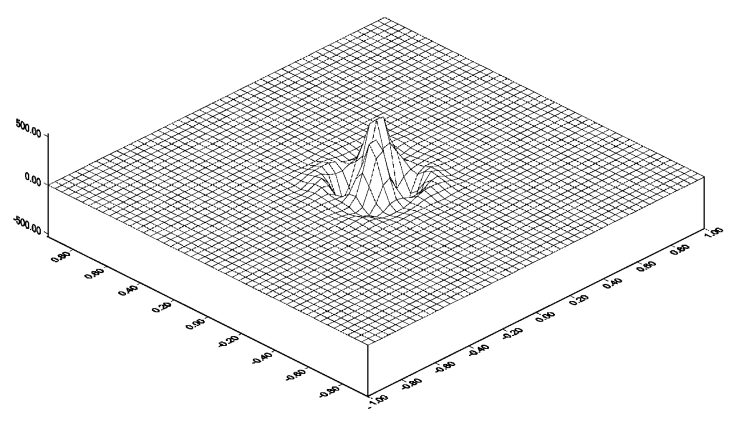

Mode $3(\mathrm{k}=3.83)$ (True)

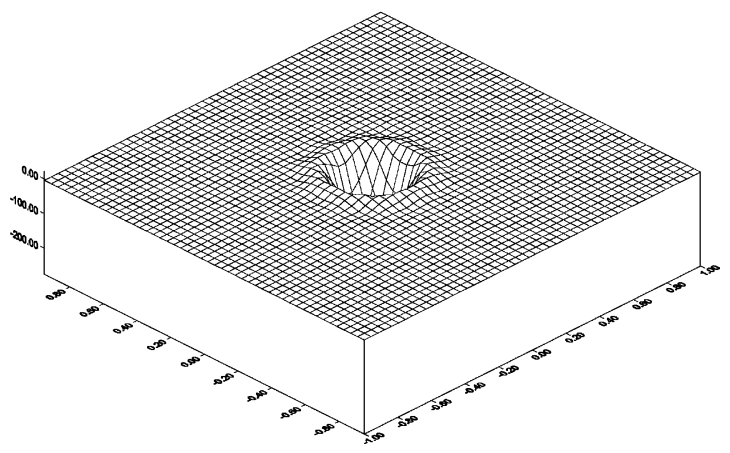

(f)

Figure 2. Continued. 
Mode $1(\mathrm{k}=1.841)$

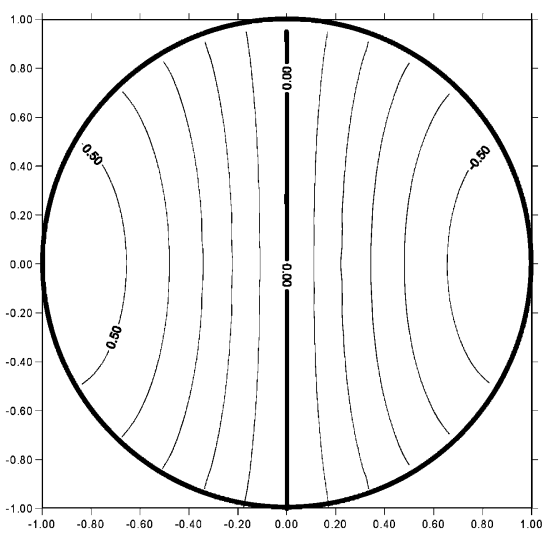

Mode $2(\mathrm{k}=3.054)$

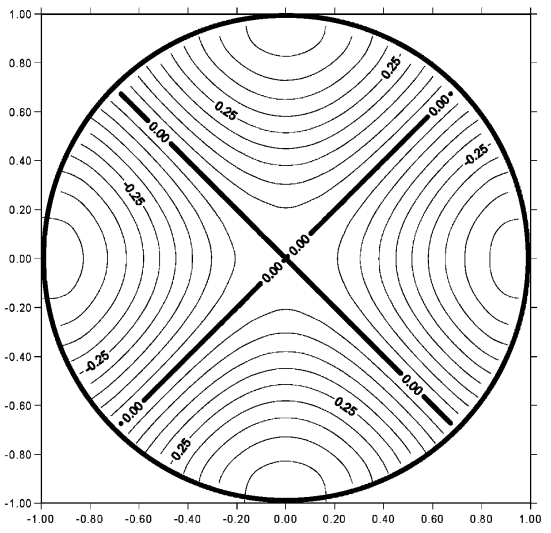

Mode $3(\mathrm{k}=3.832)$

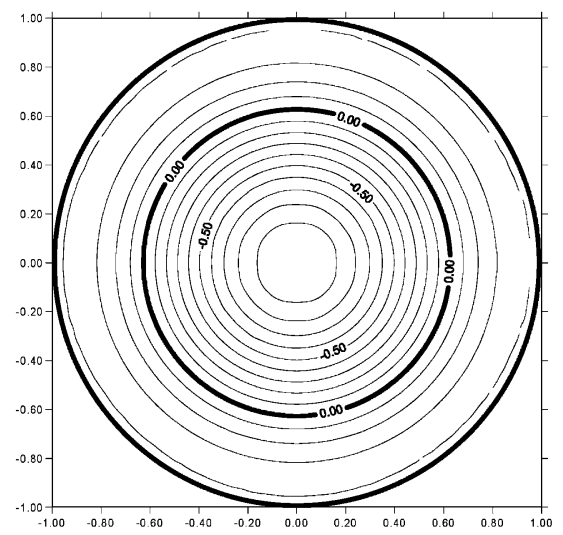

Mode $1(\mathrm{k}=1.841)$

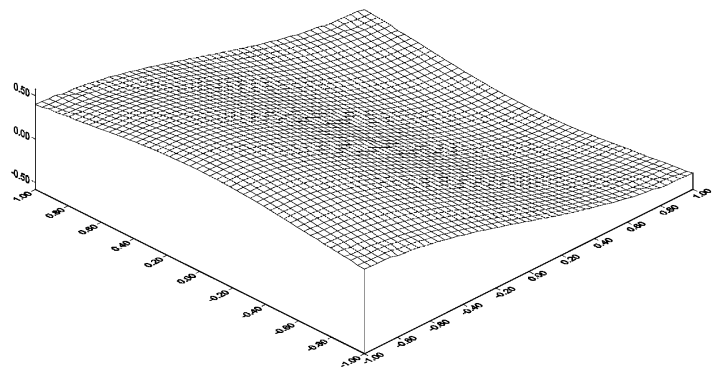

Mode $2(\mathrm{k}=3.054)$

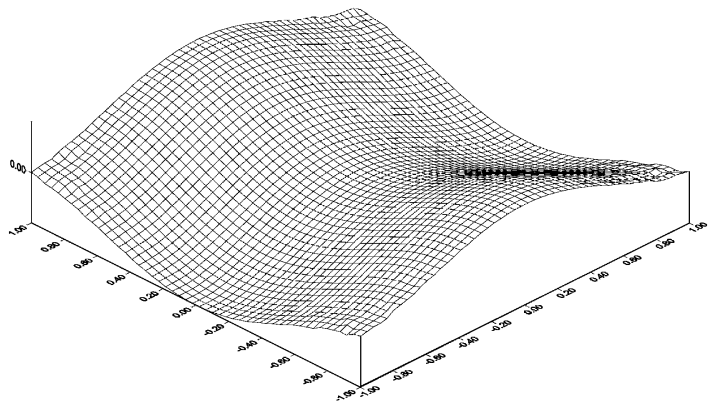

Mode $3(\mathrm{k}=3.832)$

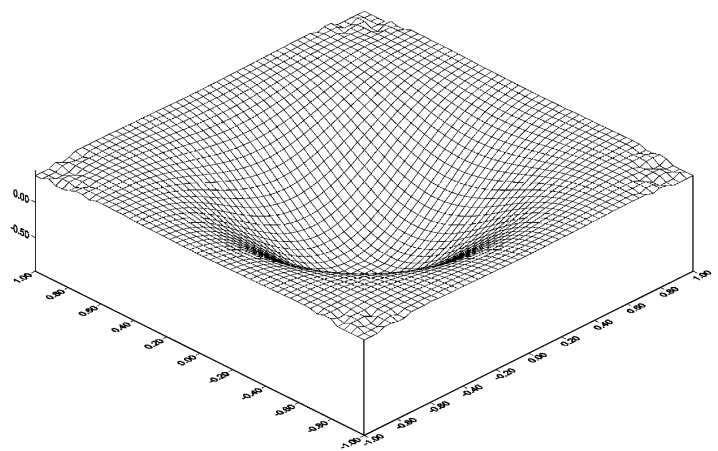

(g)

Figure 2. Continued. 
essence, we have

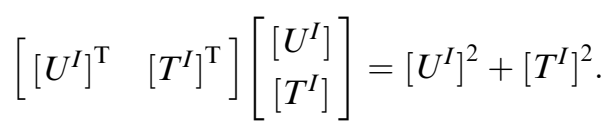

For the special case of a circular cavity in the Dirchlet problem, we can decompose $\left[U^{I}\right]$ and $\left[T^{I}\right]$ into

$$
\begin{aligned}
& {\left[U^{I}\right]=[\Phi]\left[\begin{array}{lll}
\ddots & & \\
& \lambda_{\ell} & \\
& & \ddots
\end{array}\right][\Phi]^{\mathrm{T}},} \\
& {\left[T^{I}\right]=[\Phi]\left[\begin{array}{lll}
\ddots & & \\
& \mu_{\ell} & \\
& & \ddots
\end{array}\right][\Phi]^{\mathrm{T}}}
\end{aligned}
$$

where $[\Phi]$ is a modal matrix which can be chosen as either one of the following two matrices;

$\boldsymbol{\Phi}_{1}=\frac{1}{\sqrt{2 N}}\left[\begin{array}{ccccccc}1 & \left(\mathrm{e}^{\mathrm{i} \frac{2 \pi}{2 N}}\right)^{0} & \left(\mathrm{e}^{-\mathrm{i} \frac{2 \pi}{2 N}}\right)^{0} & \cdots & \cdots & \left(\mathrm{e}^{-\mathrm{i} \frac{2(N-1) \pi}{2 N}}\right)^{0} & \left(\mathrm{e}^{\mathrm{i} \frac{2 N \pi}{2 N}}\right)^{0} \\ 1 & \left(\mathrm{e}^{\mathrm{i} \frac{2 \pi}{2 N}}\right)^{1} & \left(\mathrm{e}^{-\mathrm{i} \frac{2 \pi}{2 N}}\right)^{1} & \vdots & \vdots & \left(\mathrm{e}^{-\mathrm{i} \frac{2(N-1) \pi}{2 N}}\right)^{1} & \left(\mathrm{e}^{\left.\mathrm{i} \frac{2 N \pi}{2 N}\right)^{1}}\right. \\ 1 & \left(\mathrm{e}^{\mathrm{i} \frac{2 \pi}{2 N}}\right)^{2} & \left(\mathrm{e}^{-\mathrm{i} \frac{2 \pi}{2 N}}\right)^{2} & \vdots & \vdots & \left(\mathrm{e}^{-\mathrm{i} \frac{2(N-1) \pi}{2 N}}\right)^{2} & \left(\mathrm{e}^{\mathrm{i} \frac{2 N \pi}{2 N}}\right)^{2} \\ \vdots & \vdots & \vdots & \vdots & \vdots & \vdots \\ 1 & \left(\mathrm{e}^{\left.\mathrm{i} \frac{2 \pi}{2 N}\right)^{2 N-2}}\right. & \left(\mathrm{e}^{\left.-\mathrm{i} \frac{2 \pi}{2 N}\right)^{2 N-2}}\right. & \vdots & \vdots & \left(\mathrm{e}^{-\mathrm{i} \frac{2(N-1) \pi}{2 N}}\right)^{2 N-2} & \left(\mathrm{e}^{\mathrm{i} \frac{2 N \pi}{2 N}}\right)^{2 N-2} \\ 1 & \left(\mathrm{e}^{\mathrm{i} \frac{2 \pi}{2 N}}\right)^{2 N-1} & \left(\mathrm{e}^{\left.-\mathrm{i} \frac{2 \pi}{2 N}\right)^{2 N-1}}\right. & \cdots & \cdots & \left(\mathrm{e}^{-\mathrm{i} \frac{2(N-1) \pi}{2 N}}\right)^{2 N-1} & \left(\mathrm{e}^{\mathrm{i} \frac{2 N \pi}{2 N}}\right)^{2 N-1}\end{array}\right]_{2 N \times 2 N}$

$\Phi_{2}=$

$\frac{1}{\sqrt{2 N}}\left[\begin{array}{ccccccc}1 & 1 & 0 & \cdots & \cdots & 0 & 1 \\ 1 & \cos \left(\frac{2 \pi}{2 N}\right) & \sin \left(\frac{2 \pi}{2 N}\right) & \vdots & \vdots & \sin \left(\frac{2 \pi(N-1)}{2 N}\right) & \cos \left(\frac{2 \pi N}{2 N}\right) \\ 1 & \cos \left(\frac{4 \pi}{2 N}\right) & \sin \left(\frac{4 \pi}{2 N}\right) & \vdots & \vdots & \sin \left(\frac{4 \pi(N-1)}{2 N}\right) & \cos \left(\frac{4 \pi N}{2 N}\right) \\ \vdots & \vdots & \vdots & \vdots & \vdots & \vdots & \vdots \\ 1 & \cos \left(\frac{2 \pi(2 N-2)}{2 N}\right) & \sin \left(\frac{2 \pi(2 N-2)}{2 N}\right) & \vdots & \vdots & \sin \left(\frac{\pi(4 N-4)(N-1)}{2 N}\right) & \cos \left(\frac{\pi(4 N-4)(N)}{2 N}\right) \\ 1 & \cos \left(\frac{2 \pi(2 N-1)}{2 N}\right) & \sin \left(\frac{2 \pi(2 N-1)}{2 N}\right) & \cdots & \cdots & \sin \left(\frac{\pi(4 N-2)(N-1)}{2 N}\right) & \cos \left(\frac{\pi(4 N-2)(N)}{2 N}\right)\end{array}\right]_{2 N \times 2 N}$ 
By substituting equations (84) and (85) into (83), we obtain

$$
[C]^{\mathrm{T}}[C]=[\Phi]\left[\begin{array}{lll}
\ddots & & \\
& \left(\lambda_{\ell}^{2}+\mu_{\ell}^{2}\right) & \\
& & \ddots
\end{array}\right][\Phi]^{\mathrm{T}} .
$$

Therefore, we have the singular values of $\sqrt{\lambda_{\ell}^{2}+\mu_{\ell}^{2}}, \ell=0, \pm 1, \ldots, \pm(N-1), N$. Only the true eigenvalues $\left(\lambda_{\ell}=\mu_{\ell}=0\right)$ have dips in the figure of $\sigma_{1}$ versus $k$, i.e., the zeros of eigensolutions $\left(\mathbf{J}_{\ell}(k \rho)=0\right)$ are obtained for the Dirichlet problem.

For a circular cavity subject to the Neumann problem, we obtain the two eigenequations

$$
\begin{aligned}
& \text { Single-layer approach : } \mathbf{J}_{i}(k \rho) \mathbf{J}_{i}^{\prime}(k \rho)=0 \quad(i \text { no sum }), \\
& \text { Double-layer approach : } \mathbf{J}_{i}^{\prime}(k \rho) \mathbf{J}_{i}^{\prime}(k \rho)=0 \quad(i \text { no sum }) .
\end{aligned}
$$

The true eigensolution $\mathbf{J}_{i}^{\prime}(k \rho)=0$ satisfies both equations and the spurious eigensolutions $\mathbf{J}_{i}(k \rho)=0$ satisfies only one of equations in the single-layer potential approach for the Neumann problem. We can combine $\left[L^{I}\right]$ and $\left[M^{I}\right]$ matrices by using the updating terms to obtain an overdetermined system:

$$
[C]=\left[\begin{array}{c}
L^{I} \\
M^{I}
\end{array}\right]_{4 N \times 2 N}
$$

for the Neumann problem. Since the eigensolution is non-trivial and the rank of $[C]$ must be smaller than $2 N$. Therefore, the $2 N$ singular values for $[C]$ matrix must have at least one zero value. Based on the equivalence between the SVD technique and the least-squares method in the mathematical essence, we have

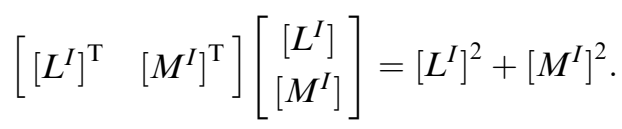

For the special case of a circular cavity of the Neumann problem, we can decompose $\left[L^{I}\right]$ and $\left[M^{I}\right]$ into

$$
\left[L^{I}\right]=[\Phi]\left[\begin{array}{lll}
\ddots & & \\
& v_{\ell} & \\
& & \ddots
\end{array}\right][\Phi]^{\mathrm{T}},
$$

\section{TABLE 5}

The former five exact eigenvalues for a two-dimensional square cavity subject to the Dirichlet and Neumann boundary conditions

\begin{tabular}{ccccccc}
\hline Problem & Eigenvalues & 1 & 2 & 3 & 4 & 5 \\
\hline Dirichlet problem & $\begin{array}{c}k_{m n}=\pi \sqrt{m^{2}+n^{2}} \\
(m, n=1,2,3, \ldots)\end{array}$ & 4.4429 & 7.0248 & 8.8858 & 9.9346 & 11.3271 \\
Neumannproblem & $\begin{array}{c}k_{m n}=\pi \sqrt{m^{2}+n^{2}} \\
(m, n=0,1,2,3, \ldots)\end{array}$ & 3.1416 & 4.4429 & 6.2831 & 7.0248 & 8.8858 \\
\hline
\end{tabular}




$$
\left[M^{I}\right]=[\Phi]\left[\begin{array}{lll}
\ddots & & \\
& \kappa_{\ell} & \\
& & \ddots
\end{array}\right][\Phi]^{\mathrm{T}} .
$$

By substituting equations (92) and (91) into equation (91), we obtain

$$
[C]^{\mathrm{T}}[C]=[\Phi]\left[\begin{array}{lll}
\ddots & & \\
& \left(v_{\ell}^{2}+\kappa_{\ell}^{2}\right) & \\
& & \ddots
\end{array}\right][\Phi]^{\mathrm{T}} .
$$

Therefore, we have the singular values of $\sqrt{v_{\ell}^{2}+\kappa_{\ell}^{2}}, \ell=0, \pm 1, \ldots, \pm(N-1), N$. Only the true eigenvalues $\left(v_{\ell}=\kappa_{\ell}=0\right)$ have dips in the figure of $\sigma_{1}$ versus $k$, i.e., the zeros of eigensolutions $\left(\mathbf{J}_{\ell}^{\prime}(k \rho)=0\right)$ are obtained for the Neumann problem.

\section{METHOD TO FILTER OUT THE SPURIOUS EIGENSOLUTIONS}

\section{Fredholm's Alternative Theorem}

(1) Nonsingular system: The equation $[H]\{g\}=\{p\}$ has a unique solution $\{g\}=[H]^{-1}\{p\}$ when the determinant of $H$ is not zero. Besides, the equation has a trivial solution $\{g\}=\{0\}$ if and only if the only continuous solution to the homogeneous equation

$$
[H]\{g\}=\{0\} .
$$

(2) Singular system: Alternatively, the equation has at least one solution if the homogeneous adjoint equation has at least one solution $\left\{\phi_{i}\right\}$ such that

$$
[H]^{\dagger}\left\{\phi_{i}\right\}=\{0\}
$$

where $[H]^{\dagger}$ is the transpose conjugate matrix of $[H]$ [24]. If the matrix $H$ is real, the transpose conjugate is equal to transpose only, i.e., $[H]^{\dagger}=[H]^{T}$. Moreover, a necessary and sufficient solvability condition [25] is that the constraint $\left(\{p\}^{\dagger}\left\{\phi_{i}\right\}=0\right)$ must be satisfied. Then, the general solution can be written as

$$
\{g\}=\{\bar{g}\}+\sum_{i=1}^{N_{r}} c_{i}\left\{\phi_{i}\right\},
$$

where $\{\bar{g}\}$ is a particular solution and $N_{r}$ is the rank of matrix $[H]$. The $c_{i}$ are arbitrary constants and $\left\{\phi_{i}\right\}$ are bases. Moreover, the particular solution is zero for the interior eigenproblems. The result implies that spurious eigenvalues are imbedded for both the Neumann and Dirichlet problems once the numerical method is chosen.

Figure 3. (a) The minimum singular value for different wave numbers by using the single-layer potential approach for the Dirichlet problem. (b) The minimum singular value for different wave numbers by using the double-layer potential approach for the Dirichlet problem. (c) The minimum singular value for different wave numbers by using the SVD updating term $\left[\begin{array}{ll}U & T\end{array}\right]$ for the Dirichlet problem. (d) The minimum singular value for different wave numbers by using the SVD updating document $\left[\begin{array}{ll}T & M\end{array}\right]$ for the Dirichlet problem. (e) The former three interior modes by using the single-layer potential approach for the Dirichlet problem. (f) The former three interior modes by using the double-layer potential approach for the Dirichlet problem. (g) The former three analytical interior modes for the Dirichlet problem. 


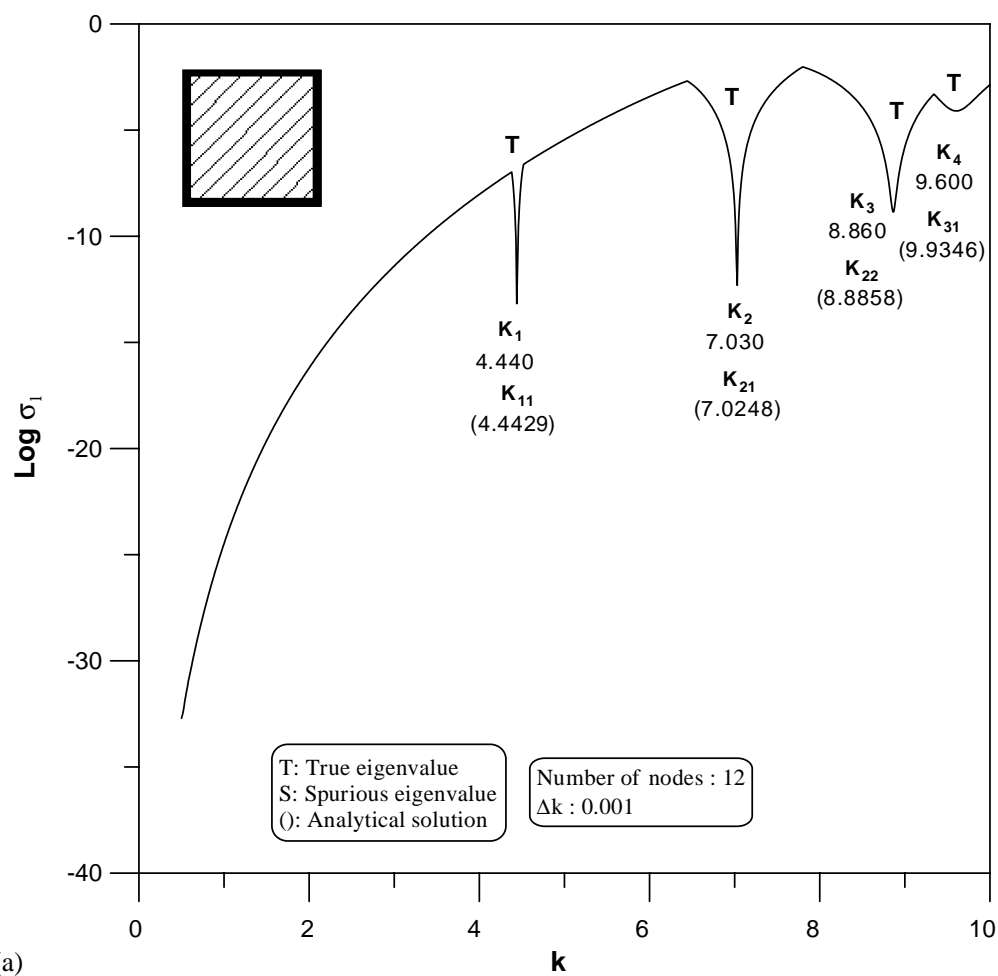

(a)

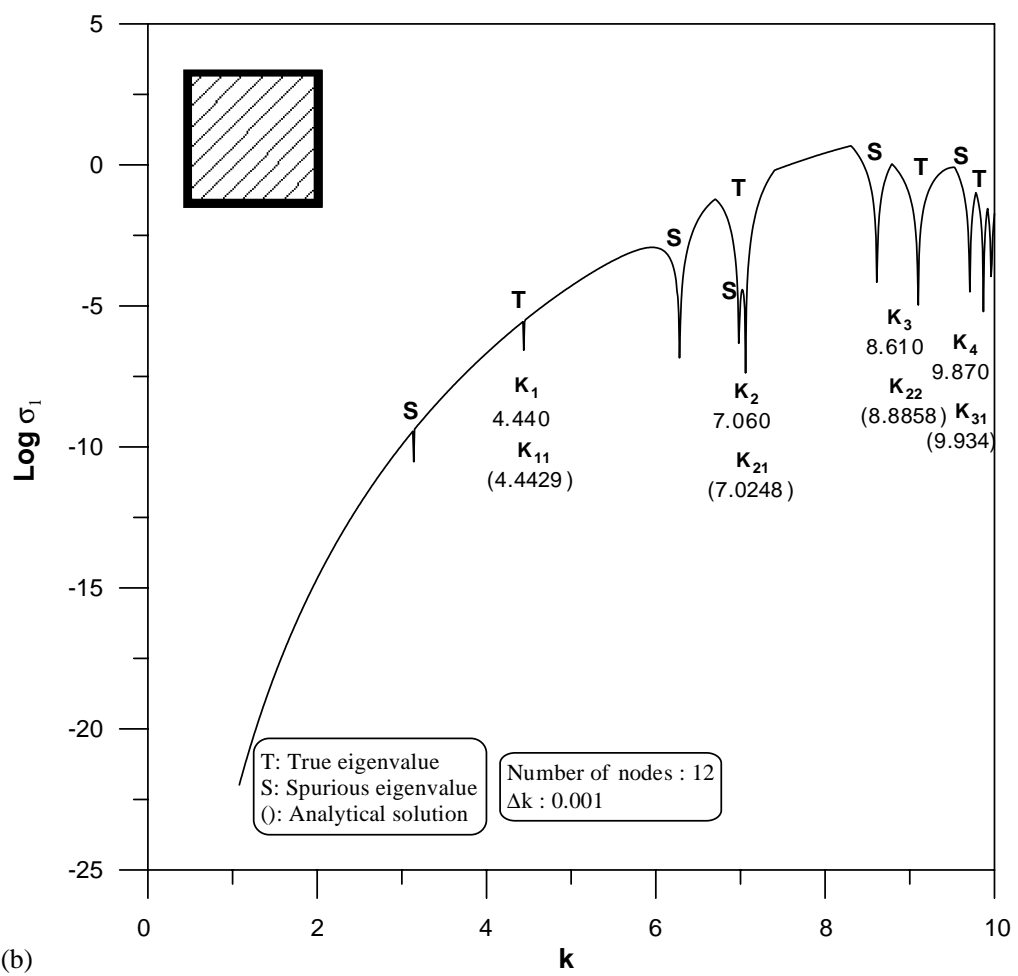



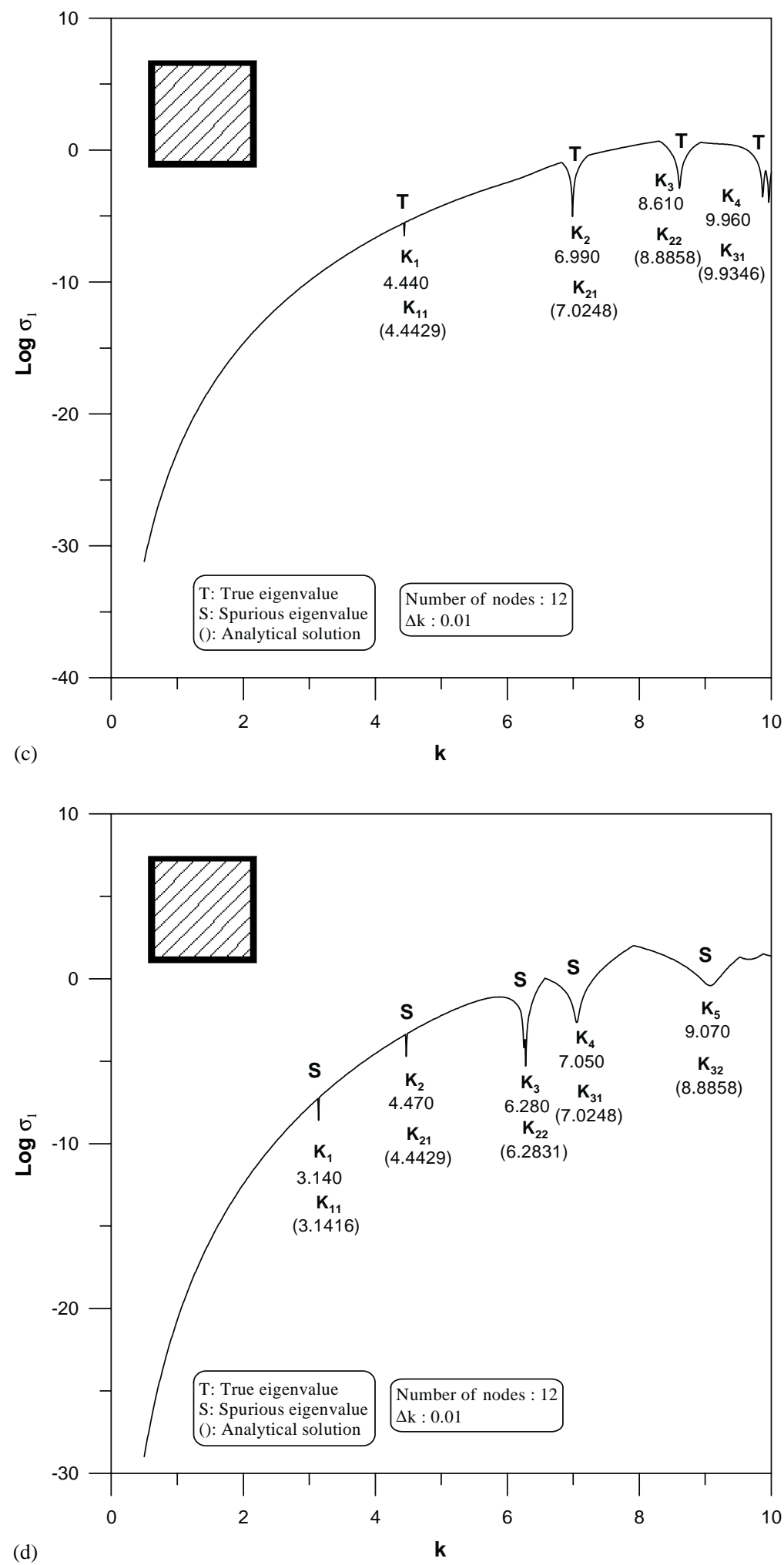

Figure 3. Continued. 

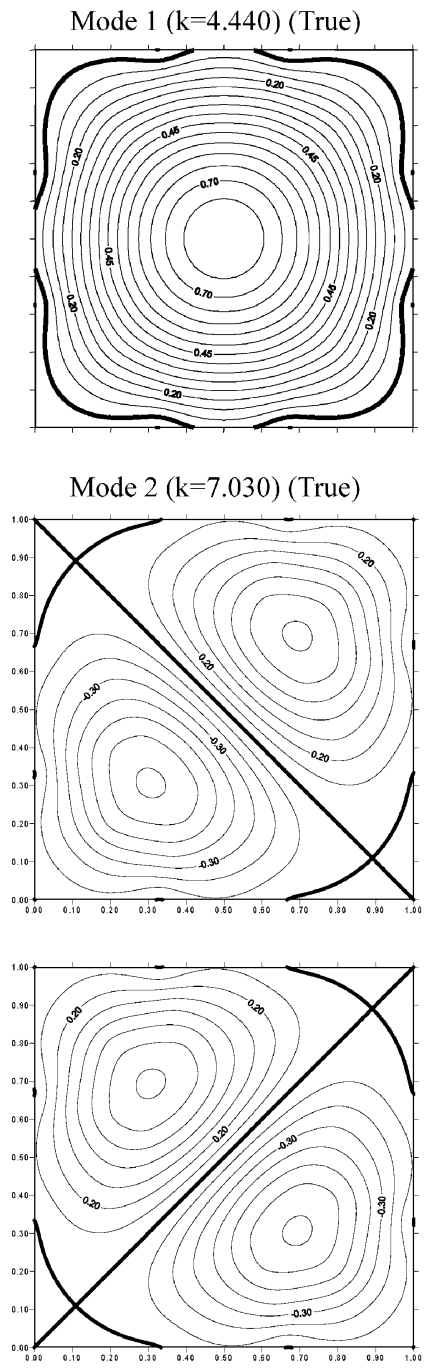

Mode $3(\mathrm{k}=8.860)$ (True)

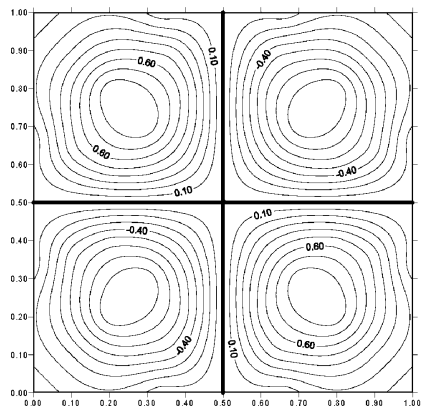

Mode $1(\mathrm{k}=4.440)$ (True)

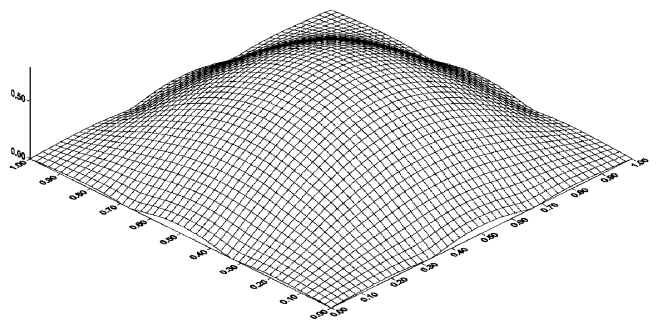

Mode $2(\mathrm{k}=7.030)$ (True)
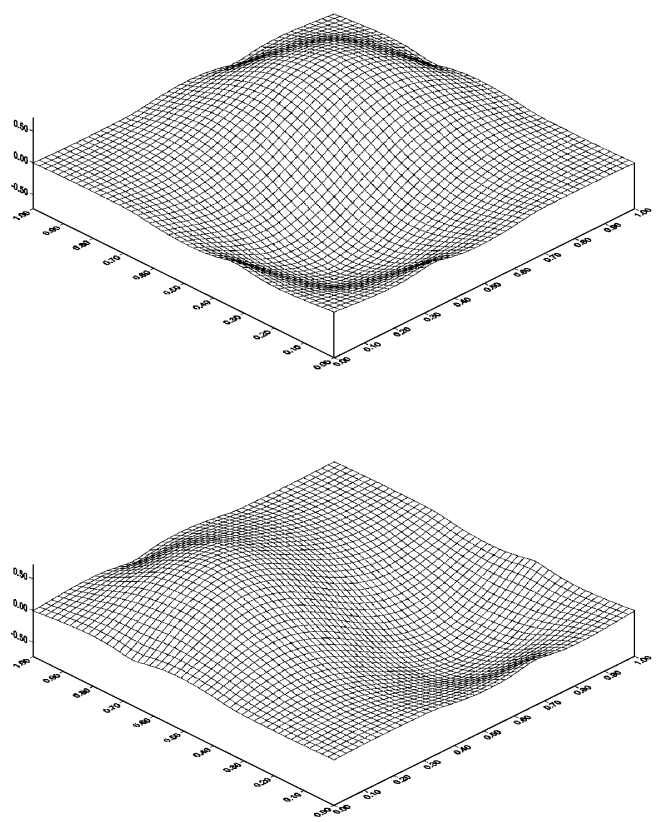

Mode $3(\mathrm{k}=8.860)$ (True)

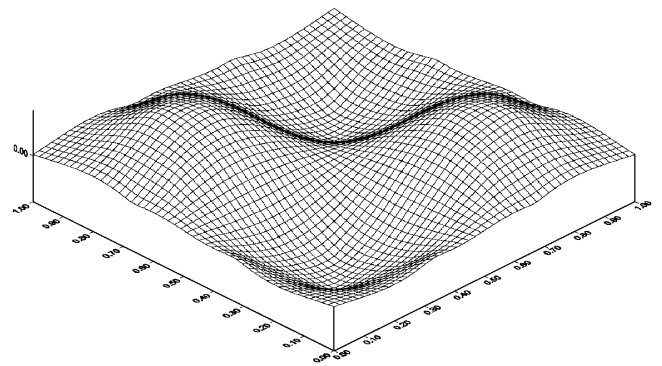

(e)

Figure 3. Continued. 


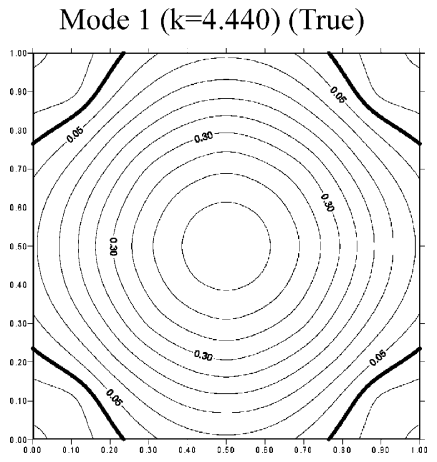

Mode $2(\mathrm{k}=7.060)$ (True)
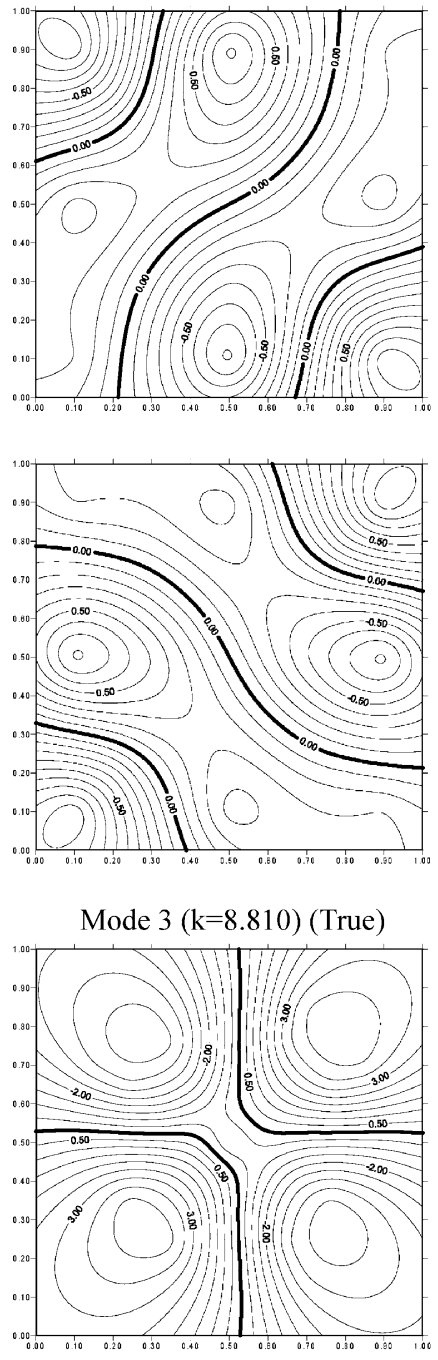

Mode $1(\mathrm{k}=4.440)$ (True)

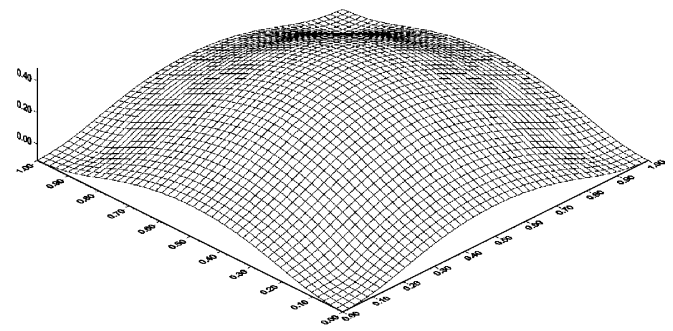

Mode $2(\mathrm{k}=7.060)$ (True)
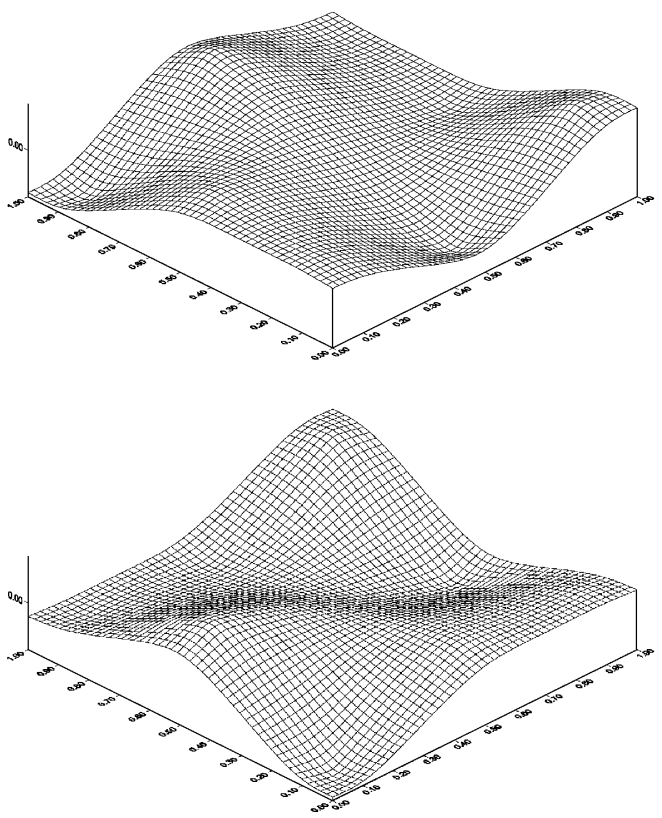

Mode $3(\mathrm{k}=8.810)$ (True)

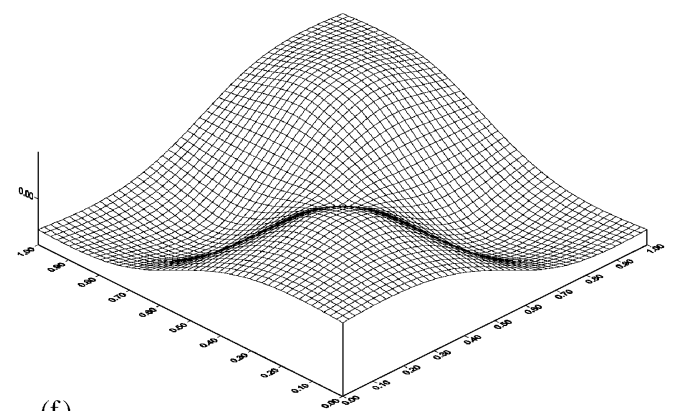

(f)

Figure 3. Continued. 
Mode $1(\mathrm{k}=4.443)$

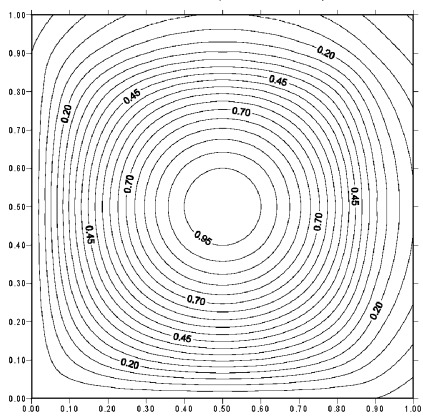

Mode $2(\mathrm{k}=7.025)$
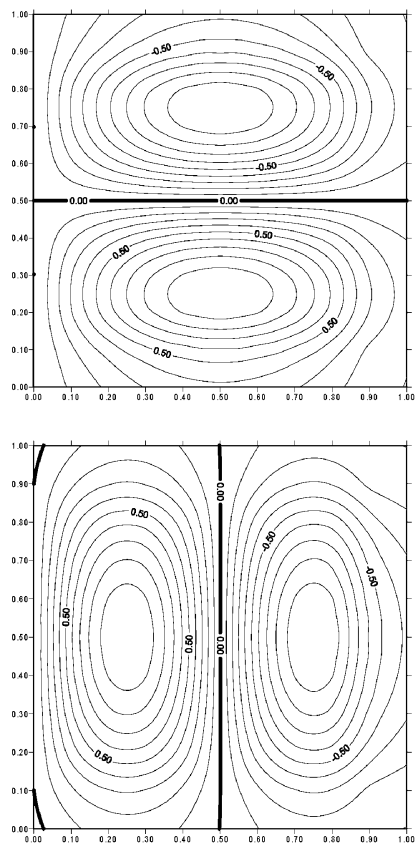

Mode $3(\mathrm{k}=8.886)$

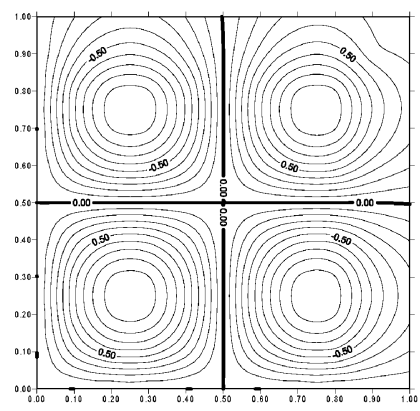

Mode $1(\mathrm{k}=4.443)$

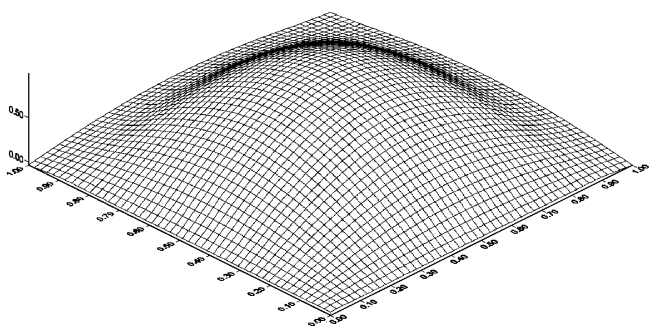

Mode $2(\mathrm{k}=7.025)$
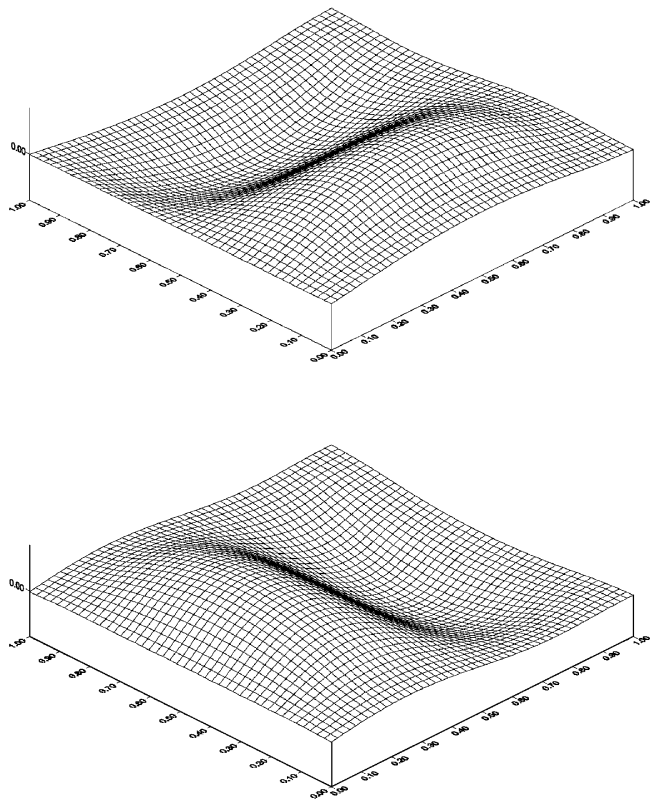

Mode $3(\mathrm{k}=8.886)$

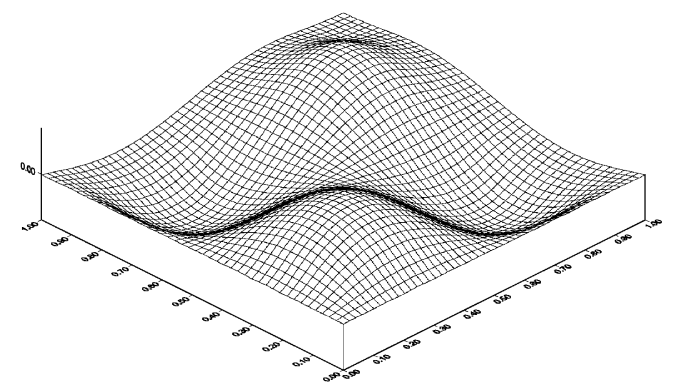

(g)

Figure 3. Continued. 
By employing the LM formulation in the direct method, we have

$$
\text { LM formulation: }\left[M^{E}\right]\{u\}=\left[L^{E}\right]\{t\}=\{p\} \text {. }
$$

Hence the eigensolution for $t$ is non-trivial, we have $\phi_{i}$ and $\left[L^{E}\right]^{\mathrm{T}} \phi_{i}=\{0\}$ such that equation (98) reduces to

$$
\left\{u^{\mathrm{T}}\right\}\left[M^{E}\right]^{\mathrm{T}}\left\{\phi_{i}\right\}=0 .
$$

Since $u$ is arbitrary for boundary excitation, we have

$$
\left[M^{E}\right]^{\mathrm{T}}\left\{\phi_{i}\right\}=\{0\} .
$$

According to Fredholm's alternative theorem in the real-matrix system, we have the homogeneous solutions in the linear algebraic equation if there exists a vector $\left\{\phi_{i}\right\}$ which satisfies

$$
\left[\begin{array}{c}
{\left[L^{E}\right]^{\mathrm{T}}} \\
{\left[M^{E}\right]^{\mathrm{T}}}
\end{array}\right]\left\{\phi_{i}\right\}=\{0\} \quad \text { or } \quad\left\{\phi_{i}\right\}^{\mathrm{T}}\left[\left[L^{E}\right] \quad\left[M^{E}\right]\right]=\{0\} .
$$

It indicates that the two matrices have the same spurious mode $\left\{\phi_{i}\right\}$ corresponding to the zero singular value. By using the SVD technique, the two matrices in equation (101) are decomposed into

$$
\begin{gathered}
{\left[L^{E}\right]^{\mathrm{T}}=\left[\Psi^{L}\right]\left[\Sigma^{L}\right]\left[\Phi^{L}\right]^{\mathrm{T}} \quad \text { or } \quad\left\{L_{j}^{E}\right\}=\sum_{j} \sigma_{j}^{L}\left\{\psi_{j}^{L}\right\}\left\{\phi_{j}^{L}\right\}^{\mathrm{T}},} \\
{\left[M^{E}\right]^{\mathrm{T}}=\left[\Psi^{M}\right]\left[\Sigma^{M}\right]\left[\Phi^{M}\right]^{\mathrm{T}} \quad \text { or } \quad\left\{M_{j}^{E}\right\}=\sum_{j} \sigma_{j}^{M}\left\{\psi_{j}^{M}\right\}\left\{\phi_{j}^{M}\right\}^{\mathrm{T}} .}
\end{gathered}
$$

By substituting equation (103) into equation (101), we have

$$
\begin{aligned}
& \sum_{j} \sigma_{j}^{L}\left\{\psi_{j}\right\}\left\{\phi_{j}\right\}^{\mathrm{T}}\left\{\phi_{i}\right\}=\{0\} \underset{\phi_{i} \cdot \overrightarrow{\phi_{j}}=\delta_{i j}}{\rightarrow} \sigma_{i}^{L}\left\{\psi_{i}\right\}=\{0\} \quad(i \text { no sum }), \\
& \sum_{j} \sigma_{j}^{M}\left\{\psi_{j}\right\}\left\{\phi_{j}\right\}^{\mathrm{T}}\left\{\phi_{i}\right\}=\{0\} \underset{\phi_{i} \cdot \overrightarrow{\phi_{j}}=\delta_{i j}}{\rightarrow} \sigma_{i}^{M}\left\{\psi_{i}\right\}=\{0\} \quad(i \text { no sum }),
\end{aligned}
$$

where $\left\{\phi_{i}\right\}$ and $\left\{\psi_{i}\right\}$ are the orthonormal bases, $\sigma_{i}^{L}$ and $\sigma_{i}^{M}$ are the zero singular values of $\left[L^{E}\right]$ and $\left[M^{E}\right]$ matrices respectively. We can easily extract out the eigensolutions since there exists the same spurious boundary mode $\left\{\phi_{i}\right\}$ corresponding to the zero singular values $\left(\sigma_{i}^{L}=\sigma_{i}^{M}=0\right)$.

Similarly, we can employ the UT formulation in the direct method,

$$
\text { UT formulation: }\left[U^{E}\right]\{t\}=\left[T^{E}\right]\{u\}=\{q\} \text {. }
$$

Since the eigensolution for $u$ is non-trivial, we have $\left\{\phi_{i}\right\}$ and $\left[T^{E}\right]^{\mathrm{T}}\left\{\phi_{i}\right\}=\{0\}$ such that equation (105) reduces to

$$
\left\{t^{\mathrm{T}}\right\}\left[U^{E}\right]^{\mathrm{T}}\left\{\phi_{i}\right\}=0
$$

Figure 4. (a) The minimum singular value for different wave numbers by using the single-layer potential approach for the Neumann problem. (b) The minimum singular value for different wave numbers by using the double-layer potential approach for the Neumann problem. (c) The minimum singular value for different wave numbers using the SVD updating term $\left[\begin{array}{ll}L & M\end{array}\right]$ for the Neumann problem. (d) The minimum singular value for different wave numbers using the SVD updating document $\left[\begin{array}{ll}U & L\end{array}\right]$ for the Neumann problem. (e) The former three interior modes by using the single-layer potential approach for the Neumann problem. (f) The former three interior modes by using the double-layer potential approach for the Neumann problem. (g) The former three analytical interior modes for the Neumann problem. 

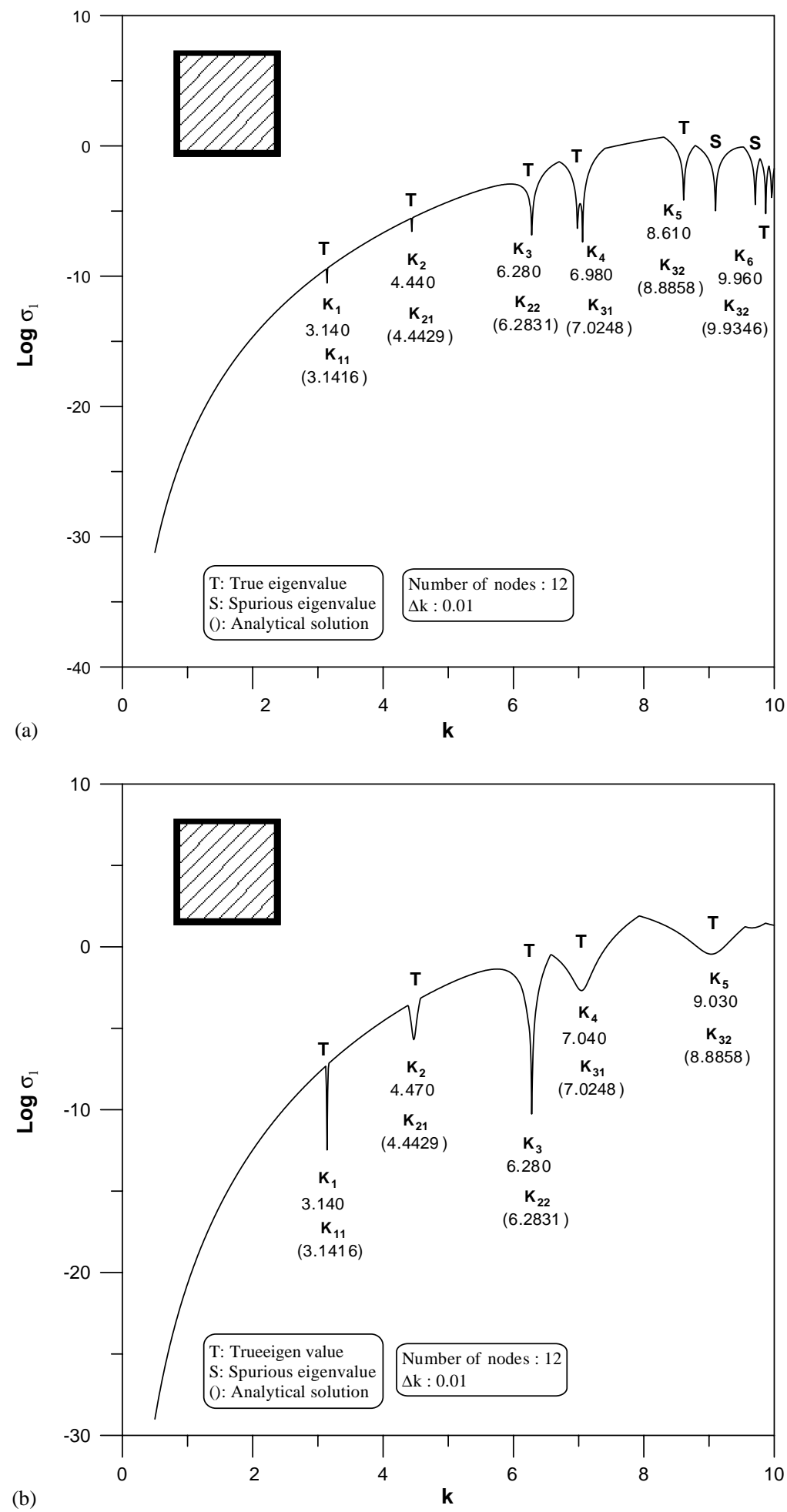

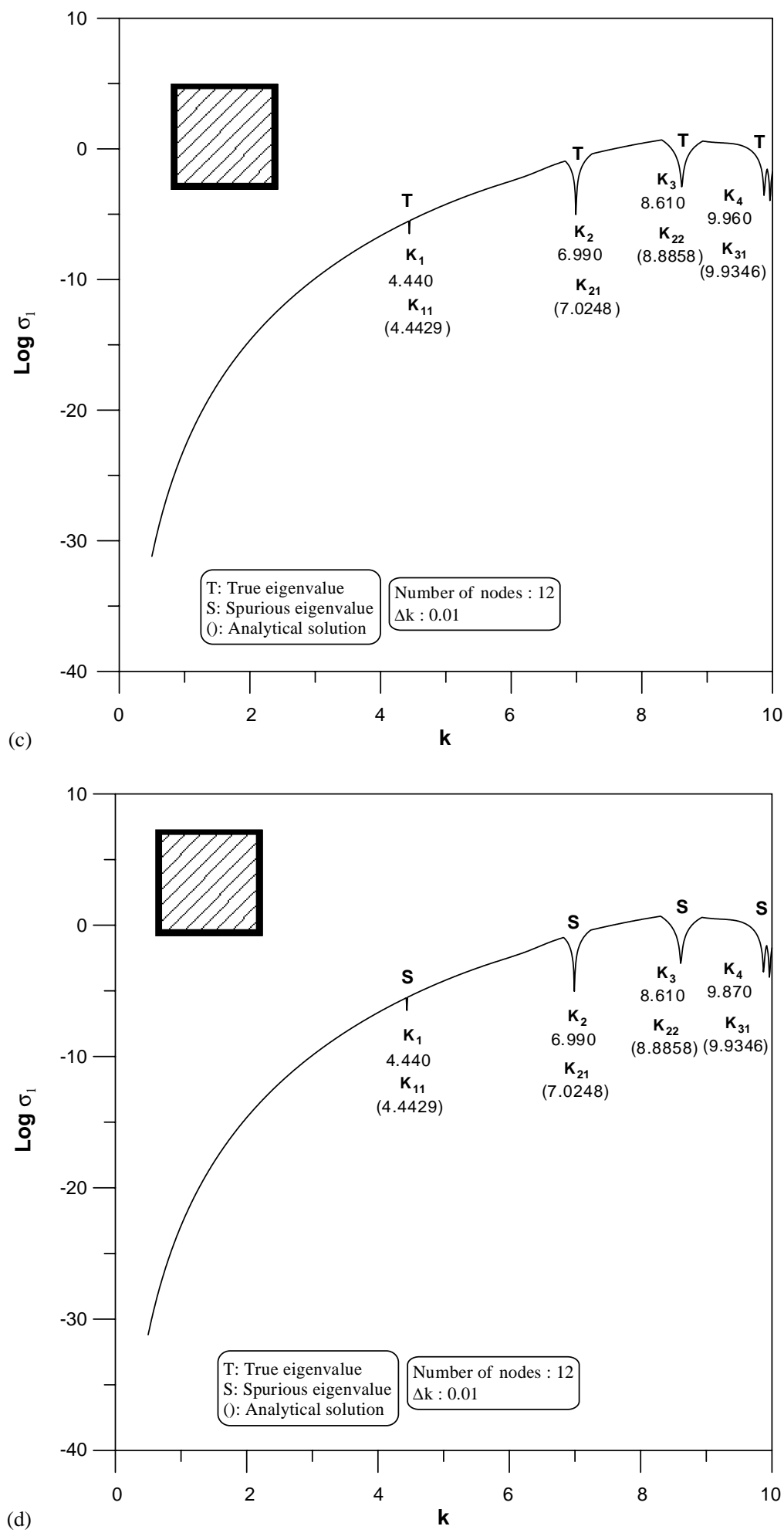

Figure 4. Continued. 

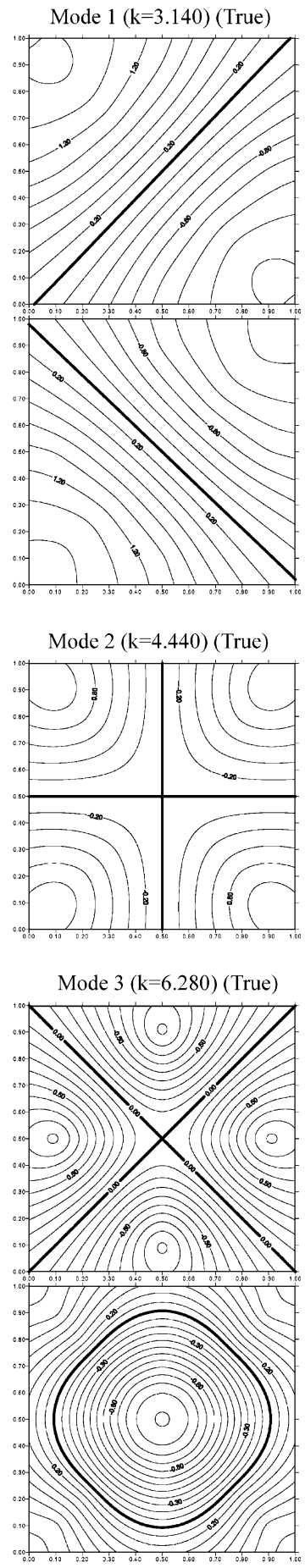

Mode $1(\mathrm{k}=3.140)$ (True)
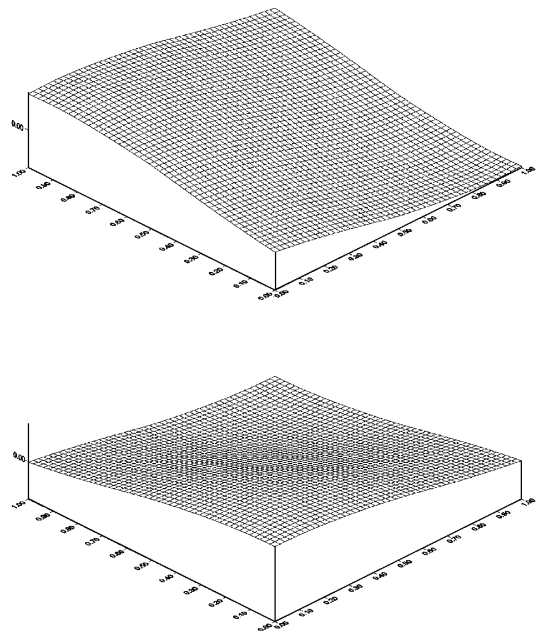

Mode $2(\mathrm{k}=4.440)$ (True)

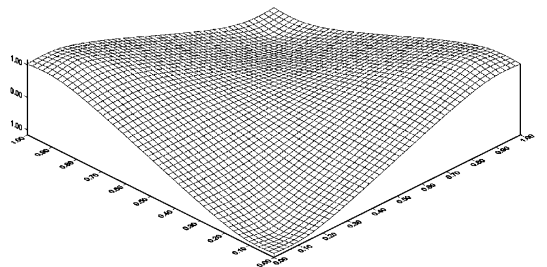

Mode $3(\mathrm{k}=6.280)$ (True)

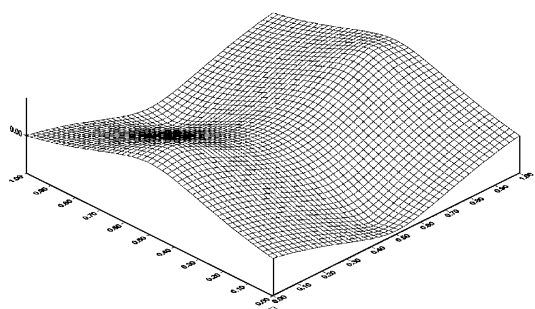

(e)

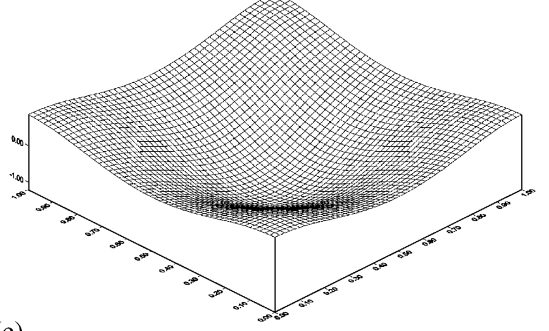

Figure 4. Continued. 


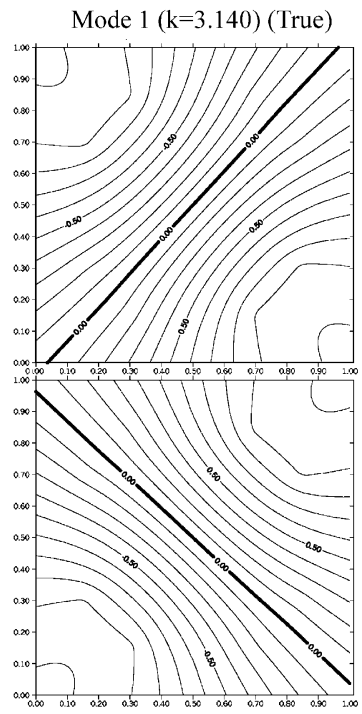

Mode $1(\mathrm{k}=3.140)$ (True)

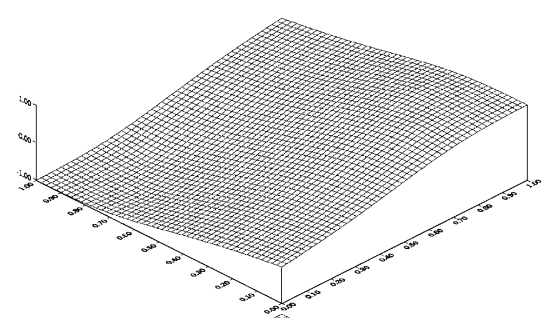

Mode $2(\mathrm{k}=4.470)$ (True)
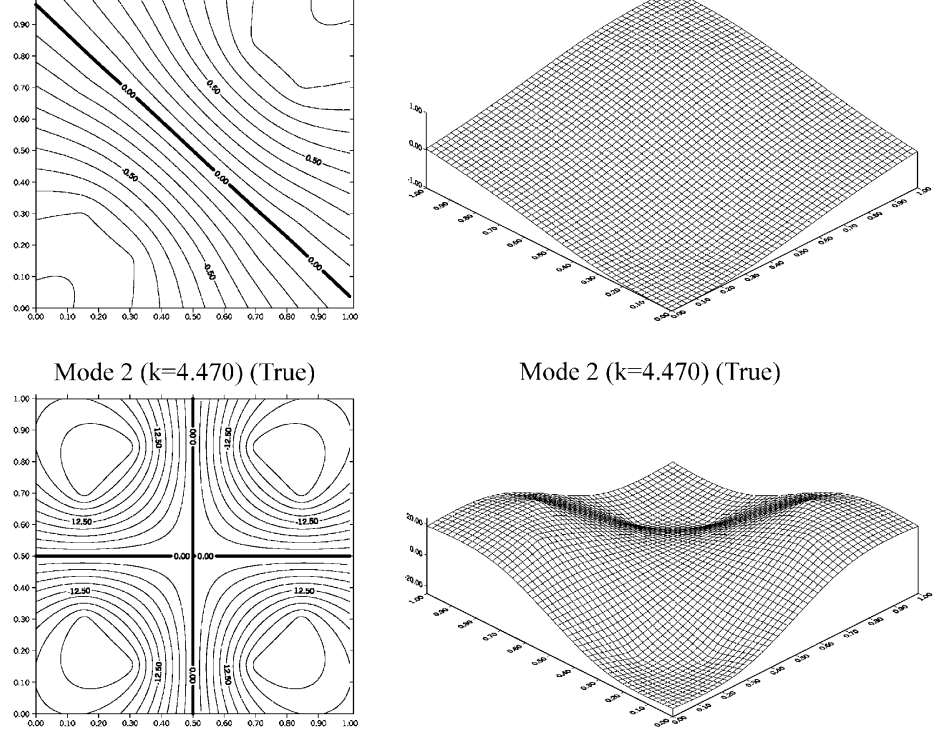

Mode $2(\mathrm{k}=4.470)$ (True)
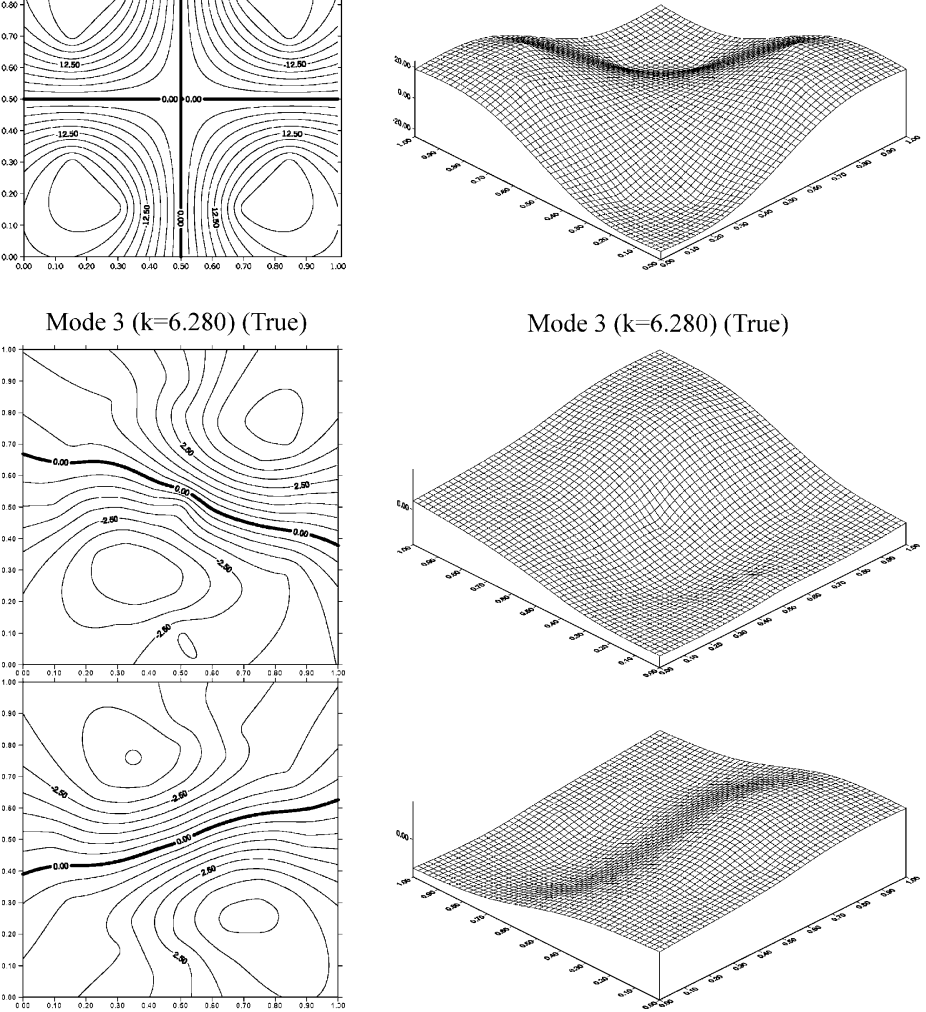

(f)

Figure 4. Continued. 


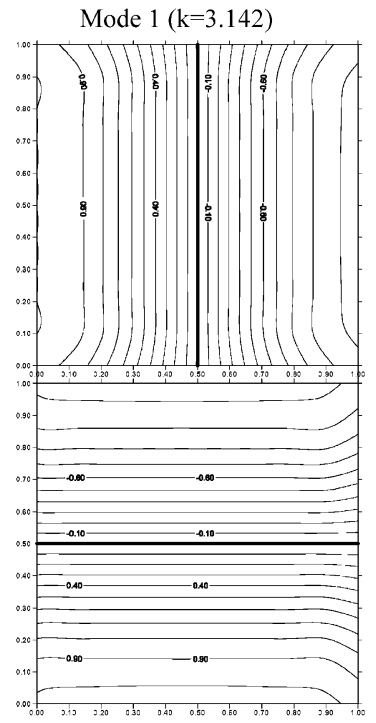

Mode $1(\mathrm{k}=3.142)$
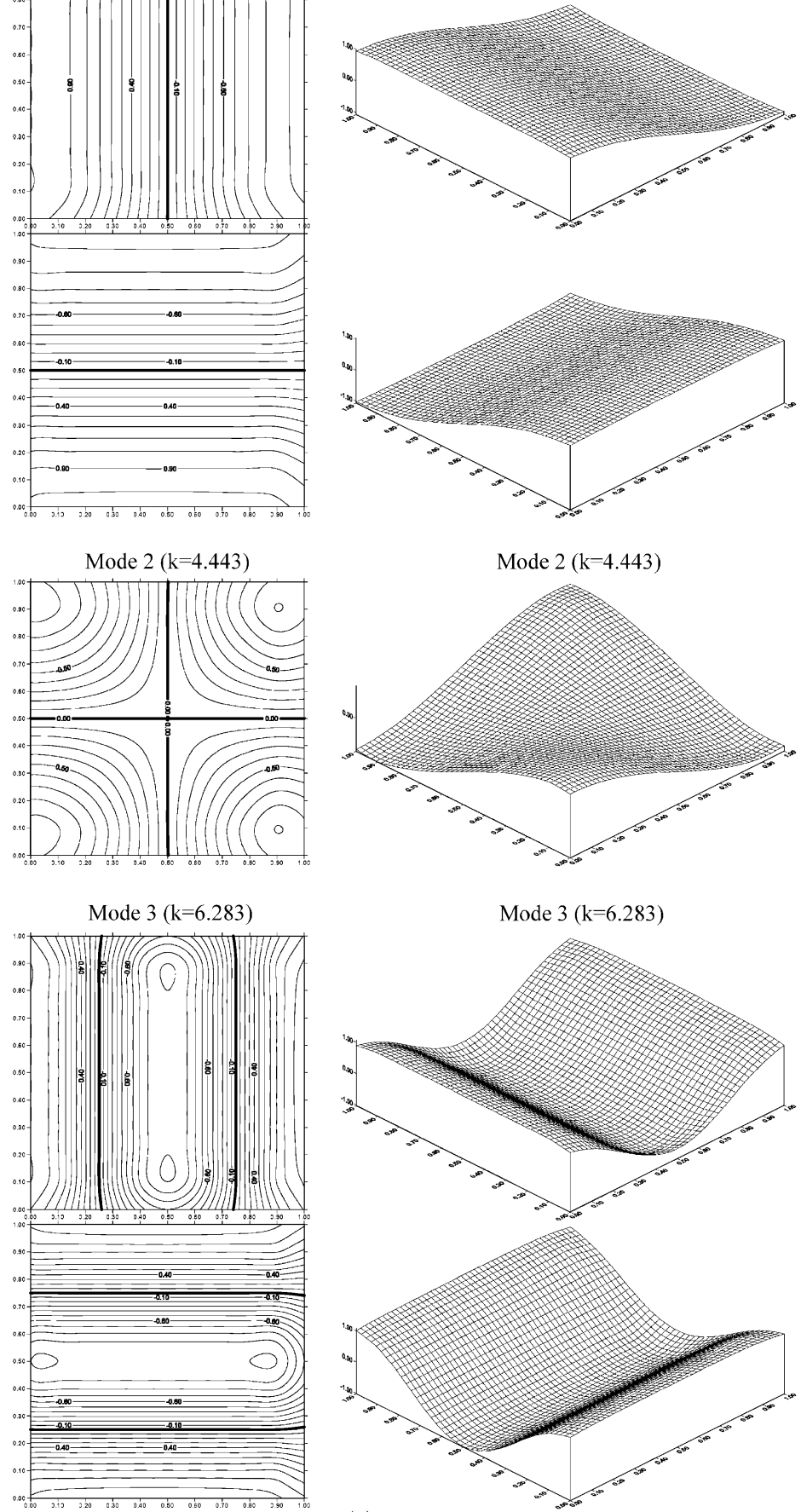

(g)

Figure 4. Continued. 
Since $\{t\}$ is arbitrary for boundary excitation, we have

$$
\left[U^{E}\right]^{\mathrm{T}}\left\{\phi_{i}\right\}=\{0\} .
$$

According to Fredholm's alternative theorem, we have the homogeneous solutions in the linear algebraic equation if there exists a vector $\left\{\phi_{i}\right\}$ which satisfies

$$
\left[\begin{array}{c}
{\left[U^{E}\right]^{\mathrm{T}}} \\
{\left[T^{E}\right]^{\mathrm{T}}}
\end{array}\right]\left\{\phi_{i}\right\}=\{0\} \quad \text { or } \quad\left\{\phi_{i}\right\}^{\mathrm{T}}\left[\left[U^{E}\right] \quad\left[T^{E}\right]\right]=\{0\} .
$$

It indicates that the two matrices have the same spurious boundary mode $\left\{\phi_{i}\right\}$ corresponding to the zero singular value. By using the SVD technique, equation (108) is expressed as

$$
\begin{aligned}
& {\left[U^{E}\right]^{\mathrm{T}}=[\Psi]\left[\Sigma^{U}\right][\Phi]^{\mathrm{T}},} \\
& {\left[T^{E}\right]^{\mathrm{T}}=[\Psi]\left[\Sigma^{\mathrm{T}}\right][\Phi]^{\mathrm{T}} .}
\end{aligned}
$$

By substituting equation (110) into equation (108), we have

$$
\begin{aligned}
& \sum_{j} \sigma_{j}^{U}\left\{\psi_{j}\right\}\left\{\phi_{j}\right\}^{\mathrm{T}}\left\{\phi_{i}\right\}=\{0\} \underset{\phi_{i} \cdot \overrightarrow{\phi_{j}}=\delta_{i j}}{\rightarrow} \sigma_{i}^{U}\left\{\psi_{i}\right\}=\{0\} \quad \text { (i no sum), } \\
& \left.\sum_{j} \sigma_{j}^{T}\left\{\psi_{j}\right\}\left\{\phi_{j}\right\}^{\mathrm{T}}\left\{\phi_{i}\right\}=\{0\} \underset{\phi_{i} \cdot \phi_{j}=\delta_{i j}}{\longrightarrow} \sigma_{i}^{T}\left\{\psi_{i}\right\}=\{0\} \quad \text { ( } i \text { no sum }\right),
\end{aligned}
$$

where $\left\{\phi_{i}\right\}$ and $\left\{\psi_{i}\right\}$ are the orthonormal bases, $\sigma_{i}^{U}$ and $\sigma_{i}^{T}$ are the zero singular values of $\left[U^{E}\right]$ and $\left[T^{E}\right]$ matrices, respectively. We can easily extract out the eigensolutions since there exists the same spurious boundary mode $\left\{\phi_{i}\right\}$ corresponding to the zero singular values $\left(\sigma_{i}^{U}=\sigma_{i}^{T}=0\right)$.

According to the relations of the matrices between the direct method and indirect method (equations (49)-(52)), we can filter out the spurious eigensolutions in the indirect method by using

$$
\left[\begin{array}{c}
{\left[L^{E}\right]^{\mathrm{T}}} \\
{\left[M^{E}\right]^{\mathrm{T}}}
\end{array}\right]\left\{\phi_{i}\right\}=\{0\} \frac{L^{E}=T^{I}}{M^{E}=M^{I}}\left[\begin{array}{c}
{\left[T^{I}\right]^{\mathrm{T}}} \\
{\left[M^{I}\right]^{\mathrm{T}}}
\end{array}\right]\left\{\phi_{i}\right\}=\{0\} .
$$

By employing the SVD technique, we obtain

$$
\begin{aligned}
& \sum_{j} \sigma_{j}^{T}\left\{\psi_{j}\right\}\left\{\phi_{j}\right\}^{\mathrm{T}}\left\{\phi_{i}\right\}=\{0\} \underset{\phi_{i} \cdot \phi_{j}=\delta_{i j}}{\rightarrow} \sigma_{i}^{T}\left\{\psi_{i}\right\}=\{0\} \quad(i \text { no sum }), \\
& \sum_{j} \sigma_{j}^{M}\left\{\psi_{j}\right\}\left\{\phi_{j}\right\}^{\mathrm{T}}\left\{\phi_{i}\right\}=\{0\} \underset{\phi_{i} \cdot \phi_{j}=\delta_{i j}}{\rightarrow} \sigma_{i}^{M}\left\{\psi_{i}\right\}=\{0\} \quad(i \text { no sum }),
\end{aligned}
$$

where $\left\{\phi_{i}\right\}$ and $\left\{\psi_{i}\right\}$ are the orthonormal bases, $\sigma_{i}^{T}$ and $\sigma_{i}^{M}$ are the zero singular values of $\left[T^{I}\right]$ and $\left[M^{I}\right]$ matrices, respectively. We can easily extract out the eigensolutions since there exists the same spurious boundary mode $\left\{\phi_{i}\right\}$ corresponding to the zero singular values $\left(\sigma_{i}^{T}=\sigma_{i}^{M}=0\right)$.

In a similar way, we have the influence matrices by using the indirect method and equation (108) is transformed to

$$
\left[\begin{array}{c}
{\left[U^{E}\right]^{\mathrm{T}}} \\
{\left[T^{E}\right]^{\mathrm{T}}}
\end{array}\right]\left\{\phi_{i}\right\}=\{0\} \frac{U^{E}=U^{I}}{T^{E}=L^{I}}\left[\begin{array}{c}
{\left[U^{I}\right]^{\mathrm{T}}} \\
{\left[L^{I}\right]^{\mathrm{T}}}
\end{array}\right]\left\{\phi_{i}\right\}=\{0\} .
$$


By using the SVD technique, we obtain

$$
\begin{aligned}
& \sum_{j} \sigma_{j}^{U}\left\{\psi_{j}\right\}\left\{\phi_{j}\right\}^{\mathrm{T}}\left\{\phi_{i}\right\}=\{0\} \underset{\phi_{i} \cdot \overrightarrow{\phi_{j}}=\delta_{i j}}{\rightarrow} \sigma_{i}^{U}\left\{\psi_{i}\right\}=\{0\} \quad(i \text { no sum }), \\
& \sum_{j} \sigma_{j}^{L}\left\{\psi_{j}\right\}\left\{\phi_{j}\right\}^{\mathrm{T}}\left\{\phi_{i}\right\}=\{0\} \underset{\phi_{i} \cdot \overrightarrow{\phi_{j}}=\delta_{i j}}{\rightarrow} \sigma_{i}^{L}\left\{\psi_{i}\right\}=\{0\} \quad(i \text { no sum }),
\end{aligned}
$$

where $\left\{\phi_{i}\right\}$ and $\left\{\psi_{i}\right\}$ are the orthonormal bases, $\sigma_{i}^{U}$ and $\sigma_{i}^{L}$ are the singular values of $\left[U^{I}\right]$ and $\left[L^{I}\right]$ matrices, respectively. We can easily extract out the eigensolutions since there exists the same spurious boundary mode $\left\{\phi_{i}\right\}$ corresponding to the zero singular values $\left(\sigma_{i}^{U}=\sigma_{i}^{L}=0\right)$.

\subsection{FILTERING OUT THE SPURIOUS EIGENSOLUTIONS BY USING SVD UPDATING DOCUMENT FOR A CIRCULAR CASE}

In solving a circular cavity problem using the double-layer potential approach, we obtain the eigenequations

$$
\begin{aligned}
& \text { Dirichlet problem : } \mathbf{J}_{i}(k \rho) \mathbf{J}_{i}^{\prime}(k \rho)=0 \quad(i \text { no sum }), \\
& \text { Neumann problem : } \mathbf{J}_{i}^{\prime}(k \rho) \mathbf{J}_{i}^{\prime}(k \rho)=0 \quad(i \text { no sum }) .
\end{aligned}
$$

The spurious eigensolution $\mathbf{J}_{i}^{\prime}(k \rho)$ are embedded in both the Dirichlet and the Neumann problems.

Based on the dual formulation, the $\left[T^{I}\right]$ and $\left[M^{I}\right]$ matrices have the same spurious eigenvalues. In order to extract the spurious eigenvalues, we can combine the $\left[T^{I}\right]$ and $\left[M^{I}\right]$ matrices by using the updating documents,

$$
[D]=\left[\left[\begin{array}{ll}
\left.T^{I}\right] & {\left[M^{I}\right]}
\end{array}\right]_{2 N \times 4 N} .\right.
$$

Similarly, we have

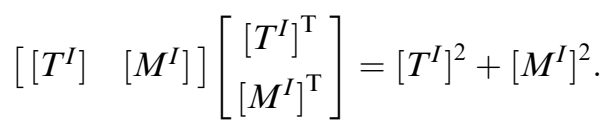

For the circular cavity, the spurious eigenvalues are both embedded in the transposes of $\left[T^{I}\right]$ and $\left[M^{I}\right]$ matrices according to equations (11)-(14). The singular values for $[D]$ must have at least one zero singular value. To determine the singular values for $[D]$, we have

$$
[D][D]^{\mathrm{T}}=[\Phi]\left[\begin{array}{lll}
\ddots & & \\
& \left(\mu_{\ell}^{2}+\kappa_{\ell}^{2}\right) & \\
& & \ddots
\end{array}\right][\Phi]^{\mathrm{T}} .
$$

By plotting the minimum singular values of $\sqrt{\mu_{\ell}^{2}+\kappa_{\ell}^{2}}, \ell=0, \pm 1, \ldots, \pm(N-1), N$ versus $k$, we can filter out the spurious eigenvalues $\left(v_{\ell}=\kappa_{\ell}=0\right)$ where dips occur, i.e., the zeros of eigensolutions $\left(\mathbf{J}_{\ell}^{\prime}(k \rho)=0\right)$ are obtained by using the double-layer potential approach.

In solving a circular cavity by using the single-layer potential approach, we obtain

$$
\text { Dirichlet problem: } \mathbf{J}_{i}(k \rho) \mathbf{J}_{i}(k \rho)=0 \quad(i \text { no sum }),
$$

Neumann problem: $\mathbf{J}_{i}(k \rho) \mathbf{J}_{i}^{\prime}(k \rho)=0 \quad(i$ no sum $)$. 
The spurious eigensolution $\mathbf{J}_{i}(k \rho)$ are embedded in both the Dirichlet and the Neumann problems.

In a similar way, we can combine the $\left[U^{I}\right]$ and $\left[L^{I}\right]$ matrices by using the updating documents,

$$
[D]=\left[\begin{array}{ll}
{\left[U^{I}\right]} & {\left[L^{I}\right]}
\end{array}\right]_{2 N \times 4 N} .
$$

Similarly, we have

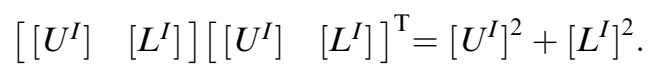

For the special case of a circular cavity, the spurious eigenvalues are embedded in the transpose of $\left[U^{I}\right]$ and $\left[L^{I}\right]$ matrices according to equations (11)-(14). The singular values for $[D]$ must have at least one zero singular value. To determine the singular values for $[D]$, we have

$$
[D][D]^{\mathrm{T}}=[\Phi]\left[\begin{array}{lll}
\ddots & & \\
& \left(\lambda_{\ell}^{2}+v_{\ell}^{2}\right) & \\
& & \ddots
\end{array}\right][\Phi]^{\mathrm{T}} .
$$

By plotting the minimum singular values of $\sqrt{\lambda_{\ell}^{2}+v_{\ell}^{2}}, \ell=0, \pm 1, \ldots, \pm(N-1), N$ versus $k$, we can filter out the spurious eigenvalues $\left(\lambda_{\ell}=v_{\ell}=0\right)$ where dips occur, i.e., the zeros of eigensolutions $\left(\mathrm{J}_{\ell}(k \rho)=0\right)$ are obtained.

All the relations between the direct and indirect methods are shown in Table 2. We can easily extract out the true and spurious eigenvalues in the indirect method or the direct method by using the SVD updating terms and documents.

\section{NUMERICAL RESULTS AND DISCUSSIONS}

Case 1: Circular cavity (Dirichlet case). A circular cavity with a radius $(\rho=1 \mathrm{~m})$ subjected to the Dirichlet boundary condition $(u=0)$ is considered. In this case, an analytical solution is available as follows:

Eigenequation:

$$
\mathbf{J}_{m}\left(k_{n} \rho\right)=0, \quad m, n=0,1,2,3 \ldots .
$$

Eigenmode:

$$
u(a, \theta)=\mathbf{J}_{m}\left(k_{n} a\right) \mathrm{e}^{\mathrm{i} m \theta}, \quad 0 \leqslant a \leqslant \rho, \quad 0 \leqslant \theta \leqslant 2 \pi, m, n=0,1,2,3, \ldots .
$$

By collocating 10 nodes on the circular boundary, two results by using the single- and double-layer potential approaches are obtained. The exact eigenvalues of a circular cavity subject to the Dirichlet boundary condition are shown in Table 3. Figure 1(a) shows the minimum singular value versus $k$ using the single-layer potential approach. The former four true eigenvalues are obtained as shown in Figure 1(a) by considering the near zero minimum singular values if only the single-layer potential method is chosen. Figure 1(b) shows the minimum singular value versus $k$ using the double-layer potential approach. The true eigenvalues occur at the positions of zeros for $\mathbf{J}_{m}\left(k_{n} \rho\right)$ while the spurious eigenvalues occur at the positions of zeros for $\mathrm{J}_{m}^{\prime}\left(k_{n} \rho\right)$ if the double-layer approach is chosen. In order to extract out the true eigenvalues, we can combine the $\left[U^{I}\right]$ and $\left[T^{I}\right]$ by using the SVD updating term. The minimum singular value versus $k$ is shown in Figure $1(\mathrm{c})$, it is found that dips occur only at the positions of true eigenvalues. In a similar way, we can combine the $\left[T^{I}\right]$ and $\left[M^{I}\right]$ by using the SVD updating document in order to filter 
out the spurious eigenvalues. The minimum singular value versus $k$ is shown in Figure 1(d), it is found that the dips occur only at the positions of spurious eigenvalues. The first three interior modes obtained by using the single- and double-layer potential approaches are shown in Figures 1(e) and 1(f), and are compared well with the analytical modes in Figure $1(\mathrm{~g})$. It is observed that the nodal line can rotate in case of the eigenvalue of multiplicity more than one. There are two independent eigenmodes with respect to the same eigenvalue. The two modes can be linearly combined by any constants and can differ by the phase lag. This is the reason why the one mode can be rotated to another mode. A similar explanation can also be found in Reference [26]. It is also found that the result of case 1 in Figure 1(e) does not match the analytical solution very well. The reason is that the amplitudes of the modes are very small since they are multiplied by a near-zero value. However, the nodal line can be identified. To demonstrate this point, the exact solutions for the mode using the present method are shown to have the near-zero solutions in Table 4. The near-zero solutions are present if the single-layer approach was applied to solve the Dirichlet problem.

Case 2: Circular cavity (Neumann case). A circular cavity with a radius $(\rho=1 \mathrm{~m})$ subjected to the Neumann boundary condition $(t=0)$ is considered. In this case, an analytical solution is available as follows:

Eigenequation:

$$
\mathbf{J}_{m}^{\prime}\left(k_{n} \rho\right)=0, \quad m, n=0,1,2,3, \ldots
$$

\section{Eigenmode:}

$$
u(a, \theta)=\mathbf{J}_{m}\left(k_{n} a\right) \mathrm{e}^{\mathrm{i} m \theta}, \quad 0 \leqslant a \leqslant \rho, 0 \leqslant \theta \leqslant 2 \pi, m, n=0,1,2,3, \ldots .
$$

By collocating 10 nodes on the circular boundary, two results by using the single- and double-layer potential approaches are obtained. The exact eigenvalues of a circular cavity subject to the Neumann boundary condition are shown in Table 3. Figure 2(a) shows the minimum singular value versus $k$ using the single-layer potential approach. The former four true eigenvalues are obtained as shown in Figrue 2(a) by considering the near-zero minimum singular values if only the single-layer potential method is chosen. Figure 2(b) shows the minimum singular value versus $k$ using the double-layer potential approach. The true eigenvalues occur at the positions of zeros for $\mathbf{J}_{m}^{\prime}\left(k_{n} \rho\right)$ while the spurious eigenvalues occur at the positions of zeros for $\mathbf{J}_{m}\left(k_{n} \rho\right)$ if the double-layer approach is chosen. In order to extract out the true eigenvalues, we combine the $\left[L^{I}\right]$ and $\left[M^{I}\right]$ by using the SVD updating term. The minimum singular value versus $k$ is shown in Figure 2(c), it is found that the dips occur only at the positions of true eigenvalues. In a similar way, we combine the $\left[U^{I}\right]$ and $\left[L^{I}\right]$ by using the SVD updating document in order to filter out the spurious eigenvalues. The minimum singular value versus $k$ is shown in Figure 2(d), it is found that the dips occur only at the positions of spurious eigenvalues. The first three interior modes obtained by using the single- and double-layer potential approaches are shown in Figure 2(e) and Figure 2(f), and are compared well with the analytical modes in Figrue 2(g) for the nodal lines. It is found that the nodal line can rotate in case of the eigenvalue of multiplicity more than one. There are two independent eigenmodes with respect to the same eigenvalue. The two modes can be linearly combined by any constants and can differ by the phase lag. This is the reason why the one mode can be rotated to another mode. A similar explanation can also be found in Reference [26]. It is also found that the result of case 2 in Figure 2(f) does not match the analytical solution very well. The reason is that the amplitudes of the modes are very small since they are multiplied by a near-zero value. However, the nodal line can be identified. To demonstrate this point, the exact solutions for the mode using the present method are shown to have the near-zero 
solutions in Table 4. The near-zero solutions are present if the double-layer approach was applied to solve the Neumann problem.

Case 3: Square cavity (Dirichlet case). A square cavity with each side of a unit length $(L=1)$ subjected to the Dirichlet boundary condition $(u=0)$ is considered. In this case, an analytical solution is available as follows:

Eigenvalues:

$$
k_{m n}=\sqrt{\left(\frac{m}{L}\right)^{2}+\left(\frac{n}{L}\right)^{2}} \pi, \quad m, n=1,2,3, \ldots
$$

Eigenmode:

$$
\sin \left(\frac{m \pi x}{L}\right) \sin \left(\frac{n \pi x}{L}\right), m, n=1,2,3, \ldots
$$

By collocating 12 nodes on the boundary, two results by using the single- and doublelayer potential approaches are obtained. The exact eigenvalues of a square cavity subject to the Dirichlet boundary condition are shown in Table 5. Figure 3(a) shows the minimum singular value versus $k$ using the single-layer potential approach. The former four true eigenvalues are obtained as shown in Figure 3(a) by considering the near-zero minimum singular values if only the single-layer potential method is chosen. Figure 3(b) shows the minimum singular value versus $k$ using the double-layer potential approach. The true eigenvalues occur at the positions of $k_{m n}=\sqrt{(m / L)^{2}+(n / L)^{2}}$ while the spurious eigenvalues occur if the double-layer approach is chosen. In order to extract out the true eigenvalues, we combine the $\left[U^{I}\right]$ and $\left[T^{I}\right]$ by using the SVD updating term. The minimum singular value versus $k$ is shown in Figure 3(c), it is found that the dips occur only at the positions of true eigenvalues. In a similar way, we combine the $\left[T^{I}\right]$ and $\left[M^{I}\right]$ by using the SVD updating document in order to filter out the spurious eigenvalues. The minimum singular value versus $k$ is shown in Figure 3(d), it is found that the dips occur only at the positions of spurious eigenvalues. The first three interior modes using the single- and double-layer approaches are shown in Figure 3(e) and Figure 3(f) and are compared with analytical modes in Figure $3(\mathrm{~g})$. For the eigenvalue of multiplicity more than one, some discrepancy for the nodal line is found since the eigensolution can be superimposed by two independent eigensolutions of the same eigenvalue.

Case 4: Square cavity (Neumann case). A square cavity with each side of a unit length $(L=1)$ subjected to the Neumann boundary condition $(t=0)$ is considered. In this case, an analytical solution is available as follows:

Eigenvalues:

$$
k_{m n}=\sqrt{\left(\frac{m}{L}\right)^{2}+\left(\frac{n}{L}\right)^{2}} \pi, \quad m, n=0,1,2,3, \ldots
$$

Eigenmode:

$$
\cos \left(\frac{m \pi x}{L}\right) \cos \left(\frac{n \pi x}{L}\right), \quad m, n=0,1,2,3, \ldots
$$

By collocating 12 nodes on the boundary, two results by using the single- and doublelayer approaches are obtained. The exact eigenvalues of a square cavity subject to the Neumann boundary condition are shown in Table 5. Figure 4(a) shows the minimum singular value versus $k$ using the single-layer potential approach. The former four true eigenvalues are obtained as shown in Figure 4(a) by considering the near-zero minimum singular values if only the single-layer potential method is chosen. Figure 4(b) shows the minimum singular value versus $k$ using the double-layer potential approach. The true 
TABLE 6

The true and spurious eigenvalues for circular and square cavities using the single-and double-layer potential approaches

\begin{tabular}{|c|c|c|c|c|c|}
\hline \multirow[t]{2}{*}{ Boundary-value problem } & \multirow[t]{2}{*}{ Eigensolution } & \multicolumn{2}{|c|}{ Circular cavity } & \multicolumn{2}{|c|}{ Square cavity } \\
\hline & & $\begin{array}{l}\text { Single-layer } \\
\text { potential } \\
\text { approach }\end{array}$ & $\begin{array}{l}\text { Double-layer } \\
\text { potential } \\
\text { approach }\end{array}$ & $\begin{array}{l}\text { Single-layer potential } \\
\text { approach }\end{array}$ & $\begin{array}{c}\text { Double-layer potential } \\
\text { approach }\end{array}$ \\
\hline \multirow[t]{4}{*}{ Dirichlet problem } & True eigensolution & $\mathbf{J}_{m}(k \rho)=0$ & $\mathbf{J}_{m}(k \rho)=0$ & $k_{m n}=\sqrt{\left(\frac{m}{L}\right)^{2}+\left(\frac{n}{L}\right)^{2}} \pi$ & $k_{m n}=\sqrt{\left(\frac{m}{L}\right)^{2}+\left(\frac{n}{L}\right)^{2}} \pi$ \\
\hline & & (Figure 1(a)) & (Figure 1(b)) & $\begin{array}{c}(m, n=1,2,3, \ldots) \\
\quad(\text { Figure } 3(\mathrm{a}))\end{array}$ & $\begin{array}{l}(m, n=1,2,3, \ldots) \\
\quad(\text { Figure } 3(\mathrm{~b}))\end{array}$ \\
\hline & Spurious eigensolution & $\mathbf{J}_{m}(k \rho)=0$ & $\mathbf{J}_{m}^{\prime}(k \rho)=0$ & $k_{m n}=\sqrt{\left(\frac{m}{L}\right)^{2}+\left(\frac{n}{L}\right)^{2}} \pi$ & $k_{m n}=\sqrt{\left(\frac{m}{L}\right)^{2}+\left(\frac{n}{L}\right)^{2}} \pi$ \\
\hline & & (Figure 1(a)) & (Figure 1(b)) & $\begin{array}{c}(m, n=1,2,3, \ldots) \\
\quad(\text { Figure } 3(\mathrm{a}))\end{array}$ & $\begin{array}{c}m, n=0,1,2,3, \ldots) \\
\quad(\text { Figure } 3(\mathrm{~b}))\end{array}$ \\
\hline \multirow[t]{4}{*}{ Neumann problem } & True eigensolution & $\mathbf{J}_{m}^{\prime}(k \rho)=0$ & $\mathbf{J}_{m}^{\prime}(k \rho)=0$ & $k_{m n}=\sqrt{\left(\frac{m}{L}\right)^{2}+\left(\frac{n}{L}\right)^{2}} \pi$ & $k_{m n}=\sqrt{\left(\frac{m}{L}\right)^{2}+\left(\frac{n}{L}\right)^{2}} \pi$ \\
\hline & & (Figure 2(a)) & (Figure 2(b)) & $\begin{array}{l}(m, n=0,1,2,3, \ldots) \\
\quad(\text { Figure } 4(\mathrm{a}))\end{array}$ & $\begin{array}{l}m, n=0,1,2,3, \ldots) \\
\quad(\text { Figure } 4(\mathrm{~b}))\end{array}$ \\
\hline & Spurious eigensolution & $\mathbf{J}_{m}(k \rho)=0$ & $\mathbf{J}_{m}^{\prime}(k \rho)=0$ & $k_{m n}=\sqrt{\left(\frac{m}{L}\right)^{2}+\left(\frac{n}{L}\right)^{2}} \pi$ & $k_{m n}=\sqrt{\left(\frac{m}{L}\right)^{2}+\left(\frac{n}{L}\right)^{2}} \pi$ \\
\hline & & (Figure 2(a)) & (Figure 2(b)) & $\begin{array}{c}(m, n=1,2,3, \ldots) \\
\quad(\text { Figure } 4(\mathrm{a}))\end{array}$ & $\begin{array}{c}(m, n=0,1,2,3, \ldots) \\
\quad(\text { Figure } 4(\mathrm{~b}))\end{array}$ \\
\hline
\end{tabular}


eigenvalues occur at the positions of $k_{m n}=\sqrt{(m / L)^{2}+(n / L)^{2}}$ while the spurious eigenvalues occur if the double-layer approach is chosen. In order to extract out the true eigenvalues, we combine the $\left[L^{I}\right]$ and $\left[M^{I}\right]$ by using the SVD updating term. The minimum singular value versus $k$ is shown in Figure 4(c), it is found that the dips occur only at the positions of true eigenvalues. In a similar way, we combine the $\left[U^{I}\right]$ and $\left[L^{I}\right]$ by using the SVD updating document in order to filter out the spurious eigenvalues. The minimum singular value versus $k$ is shown in Figure 4(d), it is found that the dips occur only at the positions of spurious eigenvalues. The former three interior modes for the single- and double-layer potential approaches are shown in Figure 4(e) and Figure 4(f), and are compared with the analytical modes in Figure $4(\mathrm{~g})$. For the eigenvalue of multiplicity more than one, some discrepancy for the nodal line is found since the eigensolution can be superimposed by two independent eigensolutions of the same eigenvalue.

\section{CONCLUSIONS}

We have developed a new meshless method by using the imaginary-part kernel. The imaginary part in the complex-valued fundamental solution was chosen as a radial basis function to approximate the solution. Although this method is very simple by using only two-point function for the influence matrices, it results in spurious eigensolutions as shown in Table 6. Two approaches, the SVD updating terms and updating documents in conjunction with dual formulation, were proposed to extract out the true eigensolutions and to filter out the spurious eigensolutions, respectively, as shown in Table 2. Both cases, circular and square cavities, subject to the Dirichlet and the Neumann boundary conditions, were demonstrated to check the validity of the meshless formulation.

\section{ACKNOWLEDGMENTS}

Financial support from the National Science Council under Grant No. NSC-90-2211-E019-006 for National Taiwan Ocean University is gratefully acknowledged.

\section{REFERENCES}

1. R. A. Gingold and J. J. Maraghan 1977 Monthly Notices of the Royal Astronomical Society 181, 375-389. Smoothed particle hydrodynamics: theory and applications to non-spherical stars.

2. P. Lancaster and K. Salkauskas 1981 Mathematics of Computation 37, 141-158. Surfaces generated by moving least-squares methods.

3. W. K. Liu, S. Jun, S. Li, J. Adee and T. Belyschko 1995 International Journal for Numerical Methods in Engineering 38, 1655-1679. Reproducing kernel particle methods for structural dynamics.

4. W. Yeih, J. T. Chen, K. H. Chen and F. C. Wong 1998 Advances in Engineering Software 29, 7-12. A study on the multiple reciprocity method and complex-valued formulation for the Helmholtz equation.

5. J. T. Chen, C. X. Hunng and K. H. Chen 1999 Computational Mechanics 24, 41-51. Determination of spurious eigenvalues and multiplicities of true eigenvalues using the real-part dual BEM.

6. J. T. Chen and F. C. Wong 1997 Engineering Analysis with Boundary Elements 20, 25-33. Analytical derivations for one-dimensional eigenproblems using dual BEM and MRM.

7. J. T. Chen and F. C. Wong 1998 Journal of Sound and Vibration 217, 75-95. Dual formulation of multiple reciprocity method for the acoustic mode of a cavity with a thin partition. 
8. S. R. Kuo, J. T. Chen and C. X. Huang 2000 International Journal for Numerical Methods in Engineering 48, 1401-1422. Analytical study and numerical experiments for true and spurious eigensolutions of a circular cavity using the real-part dual BEM.

9. N. Kamiya, E. Ando and K. Nogae 1996 Advances in Engineering Software 26, 219-227. A new complex-valued formulation and eigenvalue analysis of the Helmholtz equation by boundary element method.

10. D. Y. Liou, J. T. Chen and K. H. Chen 1999 Journal of the Chinese Institue of Civil Hydrogen Engineering 11, 89-100. A new method for determining the acoustic modes of a two-dimensional sound field. (in Chinese)

11. G. R. G. TaI and R. P. Shaw 1974 Journal of the Acoustical Society of America 56, 796-804. Helmholtz equation eigenvalues and eigenmodes for arbitrary domains.

12. A. J. NowaK and A. C. Neves, editors 1994 Multiple Reciprocity Boundary Element Method. Southampton: Comp. Mech. Publ.

13. G. De MeY 1977 Journal of the Acoustical Society of America 11, 1340-1342. A simplified integral equation method for the calculation of the eigenvalues of Helmholtz equation.

14. S. W. KANG and J. M. LeE 2000 Journal of Sound and Vibration 234, 455-470. Application of free vibration analysis of membranes using the non-dimensional dynamic influence function.

15. S. W. KANG, J. M. LeE and Y. J. KANG 1999 Journal of Sound and Vibration 221, 117-132. Vibration analysis of arbitrary shaped membranes using non-dimensional dynamic influence function.

16. S. W. KANG and J. M. LeE 2000 Journal of the Acoustical Society of America 234, 1153-1160. Eigenmode analysis of arbitrarily shaped two-dimensional cavities by the method of pointmatching.

17. J. T. Chen, S. R. Kuo, K. H. Chen and Y. C. Cheng 2000 Journal of Sound and Vibration 235 , 156-171. Comments on "Vibration analysis of arbitrary shaped membranes using nondimensional dynamic influence function".

18. J. T. Chen, C. X. Huang and F. C. Wong 2000 Journal of Sound and Vibration 230, 219-230. Determination of spurious eigenvalues and multiplicities of true eigenvalues in the dual multiple reciprocity method using the singular value decomposition technique.

19. J. T. Chen, M. H. Chang, I. L. Chung and Y. C. Cheng 2002 Journal of the Acoustical Society of America 111, 33-36. Comments on "Eigenmode analysis of arbitrarily shaped twodimensional cavities by the method of point matching."

20. G. H. Golub and C. F. VAn Loan 1989 Matrix Computations. Baltimore: The Johns Hopkins University Press; second edition.

21. J. L. Goldberg 1991 Matrix Theory with Applications, New York: McGraw-Hill.

22. J. T. Chen and H. K. Hong 1999 Transactions of the American Society of Mechanical Engineers, Applied Mechanics Review 52, 17-33. Review of dual integral representations with emphasis on hypersingular integrals and divergent series.

23. J. T. Chen and K. H. Chen 1998 Engineering Analysis with Boundary Elements 21, 105-116. Dual integral formulation for determining the acoustic modes of a two-dimensional cavity with a degenerate boundary.

24. H. T. Davis and K. T. Thomson 2000 Linear Algebra and Linear Operators in Engineering with Applications in Mathematica, Vol. 3. New York: Acadamic Press.

25. I. Stakgold 1979 Green's Functions and Boundary Value Problems. New York: John Wiley \& Sons.

26. J. T. Chen, K. H. Chen and S. W. Chyunan 1999 Applied Acoustics 57, 293-325. Numerical experiments for acoustic modes of a square cavity using the dual boundary element method. 